\title{
Differential Equations and Boundary Conditions for Rarefied Polyatomic Gases
}

\author{
H. Vestner \\ Institut für Theoretische Physik der Universität Erlangen-Nürnberg, Erlangen
}

(Z. Naturforsch. 28 a, 1554-1570 [1973] ; received 7 July 1973)

\begin{abstract}
Constitutive laws and differential equations for macroscopic variables in a polyatomic gas are derived from moment equations of the Waldmann-Snider equation. Constitutive laws are stated for the friction pressure tensor, the translational and rotational heat fluxes and for the tensor polarization flux. Differential equations are given for the temperature, for the difference between translational and rotational temperatures, for the flow velocity and for the tensor polarization. Boundary conditions for these four moments are obtained by Waldmanns method from the entropy production at the interface between the gas and a solid body. In particular, these are a temperature jump condition, a velocity slip condition, and equations which determine the values at the wall of the tensor polarization and of the difference between translational and rotational temperatures.
\end{abstract}

Nonequilibrium processes in a dilute polyatomic gas can be described by the quantum mechanical kinetic equation due to Waldmann and Snider ${ }^{1}$. Interest has focussed on alignment phenomena which give information on the noncentral part of the interaction potential between the gas molecules. Prominent examples are the Senftleben-Beenakker effect of viscosity and of thermal conductivity ${ }^{2}$, the flow ${ }^{3}$ and heat-flow birefringence ${ }^{4}$, and the depolarized Rayleigh light scattered by a polyatomic gas $^{5}$.

In a rarefied gas the mean free path of a molecule is no longer negligibly small compared with macroscopic lengths of the measuring device. Then the collisions of the molecules with the walls become important besides the intermolecular collisions. Up to now there is no general kinetic theory for this case where both types of collisions are of equal importance. Mostly the problem is attacked in the following way: Away from the boundary the behaviour of the gas is described by a kinetic equation which contains only the binary molecular collisions, i.e. the Boltzmann equation for monatomic gases or the Waldmann-Snider equation for polyatomic gases. The behaviour of the gas near the boundary is characterized by a condition which relates the distribution function of the molecules leaving the wall with that of the molecules approaching the wall. The scattering kernel involved contains the gas-wall collisions. For monatomic gases this approach traces back to Maxwell, later on it has been

Reprint requests to Dr. H. Vestner, Institut für Theoretische Physik der Universität Erlangen-Nürnberg, D-8520 Erlangen, Glückstraße 6. worked out by many authors ${ }^{6}$. A first attempt for a polyatomic gas has been made by Halbritter ${ }^{7}$ and by Borman et al. ${ }^{8}$. They were especially interested in the polarizations created by gas-wall collisions.

If a condition for the distribution function at the wall is known many problems in highly rarefied gases can be treated 6,8 , furthermore boundary conditions for mean values can then be derived ${ }^{6,7}$. This is of importance for the region between the hydrodynamic and the Knudsen regime where one deals with the solution of differential equations and boundary conditions for macroscopic variables.

A general method for the derivation of phenomenological boundary conditions is due to Waldmann ${ }^{9}$. Starting point is the entropy production at the interface between two media. This interfacial entropy production consists of a discontinuity of the entropy fluxes at the dividing surface. By the use of conservation laws at the surface the interfacial entropy production is transformed into an integral over independent "fluxes" and "forces". In analogy to the theory of irreversible processes linear relations between "fluxes" and "forces" are set up, and these are the boundary conditions ${ }^{9}$. Such boundary conditions have been derived for a treatment of thermal force ${ }^{10}$, of condensation and evaporation ${ }^{11}$, of Knudsen corrections for the Senftleben-Beenakker effect of viscosity 12,13 and of thermomagnetic pressure difference ${ }^{12,13,14}$.

For a solution of flow problems in rarefied gases the condition for tangential velocity at the wall is of crucial importance. There are different types of slip effects, a mechanical, a thermal, and a polarization induced slip ("thermomagnetic slip"15). Slip 
effects in rarefied polyatomic gases in a magnetic field show up most clearly in thermomagnetic pressure difference. This experiment has been proposed in 1967 by Waldmann ${ }^{15}$ and has been done for the first time in 1972 for $\mathrm{N}_{2}$ by Hulsman et al. ${ }^{16}$. Its investigation allows the determination of the slip contributions to the thermomagnetic torque ${ }^{17}$; the bulk contributions are due to thermal stresses and have been calculated by Levi et al. ${ }^{18}$. The importance of slip for an explanation of the torque was first stressed by Waldmann 15 who introduced to this end thermomagnetic slip.

In this paper the near-continuum behaviour of a rarefied gas shall be described by constitutive laws, differential equations and boundary conditions for macroscopic variables. The nonequilibrium state of the gas of rotating molecules shall be characterized by the following quantities: The number density, the translational and rotational temperatures, the flow velocity, the translational and rotational heat fluxes, the pressure tensor, the tensor polarization and the tensor polarization flux. For most gases the tensor polarization and its flux are the dominant types of alignment ${ }^{2}$. By an application of the moment method 19 to the linearized Waldmann-Snider equation a set of coupled partial differential equations can be derived for the variables considered here. These transport relaxation equations are stated in the first part of this paper. They contain constitutive laws for the friction pressure tensor, for the heat fluxes and for the tensor polarization flux. All types of Burnett terms are included, e.g. thermal stresses are due to a first order derivative of the translational heat flux. This compares with second order Chapman-Enskog equations obtained by Levi et al. ${ }^{20}$.

For a steady state situation differential equations are given for the velocity, the temperatures and the tensor polarization. The Fourier equation for the temperature and the Navier-Stokes equation for the velocity contain modifications due to the polyatomic nature of the gas.

The second part of the paper is devoted to the derivation of boundary conditions from the entropy production at the interface between the polyatomic gas and a solid body. First, the entropy flux within the gas is calculated from the special expansion of the distribution function used in the first part. Then the interfacial entropy production is worked out, and finally the boundary conditions are stated for the flow velocity, the temperatures and the tensor polarization. This method supplies us with the most general form of boundary conditions which is compatible with thermodynamics. But it gives no quantitative results, a set of phenomenological parameters is introduced. It is a difficult task to relate these parameters to the interaction potential between a gas molecule and the wall. This problem will not be attacked here.

\section{Differential Equations and Boundary Conditions}

\section{§1. A special system of transport relaxation equations}

The nonequilibrium state of a dilute gas is described by the one-particle distribution operator which obeys the quantum mechanical kinetic equation due to Waldmann and Snider ${ }^{1}$. By an application of the moment method 19 to the linearized Waldmann-Snider equation an infinite set of transport relaxation equations for the nonequilibrium averages is obtained. In practice only a finite number of these macroscopic variables is taken into account. Throughout this paper the nonequilibrium state of a gas of rotating molecules shall be characterized by the following quantities: The density $n$, the translational and rotational temperatures $T_{\text {trans }}$ and $T_{\text {rot }}$, the mean velocity $\boldsymbol{v}$, the translational and rotational heat fluxes $\boldsymbol{q}_{\text {trans }}$ and $\boldsymbol{q}_{\text {rot }}$, and the symmetric trace ess part $\overline{\mathbf{p}}$ of the pressure tensor. Furthermore, for a description of the alignments created by collisions the tensor polarization $\mathbf{a}$ and its flux $\underline{\mathbf{b}}$ are included. The tensor polarization is the nonequilibrium average of a symmetric traceless second rank tensor built up from the (dimensionless) rotational angular momentum $\boldsymbol{J}$ of a molecule

$$
\mathbf{a}=\langle\boldsymbol{\Phi}\rangle, \quad \boldsymbol{\Phi}=\sqrt{15 / 2} R\left(J^{2}\right) \bar{J} \boldsymbol{J} .
$$

The function $R\left(J^{2}\right)$ is normalized with respect to the equilibrium distribution:

$$
\left\langle R^{2} J^{2}\left(J^{2}-\frac{3}{4}\right)\right\rangle_{0}=1 .
$$

Since here only the formal str ucture of the transport relaxation equations is of interest $R\left(J^{2}\right)$ has not to be specified. For a treatment of the SenftlebenBeenakker effect of viscosity often

$$
R\left(J^{2}\right)=\left(\left\langle J^{2}\left(J^{2}-\frac{3}{4}\right)\right\rangle_{0}\right)^{-1 / 2}
$$

is chosen ${ }^{19}$, whereas in connection with birefringence 


$$
R\left(J^{2}\right)=\left(J^{2}-\frac{3}{4}\right)^{-1}\left(\left\langle J^{2}\left(J^{2}-\frac{3}{4}\right)^{-1}\right\rangle_{0}\right)^{-1 / 2}
$$

has to be used ${ }^{3,4}$.

The tensor polarization flux is a third rank tensor,

$$
b_{\lambda, \mu v}=\frac{\sqrt{3}}{c_{0}}\left\langle c_{\lambda} \Phi_{\mu v}\right\rangle
$$

$c_{\lambda}$ is the molecular velocity, $c_{0}=\sqrt{\frac{3 k T_{0}}{M}}, M$ is the mass of a molecule, $T_{0}$ the equilibrium temperature, $k$ is Boltzmann's constant. The fact that the tensor $c_{\lambda} \Phi_{\mu \nu}$ has not been decomposed into its three irreducible components 19,21

$$
\Phi_{\mu}=\Phi_{\mu \lambda} c_{\lambda}, \Psi_{\mu \nu}=\Phi_{\mu \lambda} \varepsilon_{v \lambda \varkappa} c_{\varkappa}, \Phi_{\mu \nu \lambda}=\bar{c}_{\lambda} \Phi_{\mu \nu}
$$

implies the assumption that the relaxation coefficients of the corresponding averages are equal. This is well founded for small nonsphericity of the molecular interaction potential ${ }^{21}$.

The following transport relaxation equations are obtained:

$$
\begin{aligned}
& \partial n / \partial t+n_{0} \boldsymbol{\nabla} \cdot \boldsymbol{v}=0, \\
& n_{0} \frac{3}{2} k \frac{\partial T_{\text {trans }}}{\partial t}+\boldsymbol{\nabla} \cdot\left(\boldsymbol{q}_{\text {trans }}+p_{0} \boldsymbol{v}\right)+n_{0} \frac{3}{2} k \omega_{0}\left(T_{\text {trans }}-T_{\text {rot }}\right)=0, \\
& n_{0} c_{\mathrm{rot}}^{(0)} \frac{\partial T_{\mathrm{rot}}}{\partial t}+\boldsymbol{\nabla} \cdot \boldsymbol{q}_{\mathrm{rot}} \quad-n_{0} \frac{3}{2} k \omega_{0}\left(T_{\text {trans }}-T_{\mathrm{rot}}\right)=0 \\
& n_{0} M \frac{\partial \boldsymbol{v}}{\partial t} \quad+\boldsymbol{\nabla}\left(n k T_{0}+n_{0} k T_{\text {trans }}\right)+\boldsymbol{\nabla} \cdot \overline{\mathbf{p}}=0, \\
& \frac{\partial}{\partial t} \boldsymbol{q}_{\text {trans }} \quad+{ }_{2}^{5} p_{0} \frac{k}{M}\left(\boldsymbol{\nabla} T_{\text {trans }}+\frac{2}{5} \frac{T_{0}}{p_{0}} \boldsymbol{\nabla} \cdot \overline{\mathbf{p}}\right)+\omega_{\mathrm{t}} \boldsymbol{q}_{\text {trans }}+\omega_{\mathrm{tr}} \boldsymbol{q}_{\mathrm{rot}}+\omega_{\mathrm{bt}} / \frac{\sqrt{5}}{6} p_{0} c_{0} \boldsymbol{B}=0, \\
& \frac{\partial}{\partial t} \boldsymbol{q}_{\text {rot }} \quad+{ }_{5}^{3} \frac{c_{\mathrm{r}}}{c_{\mathrm{t}}}\left[{ }_{2}^{5} p_{0} \frac{k}{M} \boldsymbol{\nabla} T_{\text {rot }} \quad+\omega_{\text {tr }} \boldsymbol{q}_{\text {trans }}+\omega_{\mathrm{r}} \boldsymbol{q}_{\text {rot }}+\omega_{\mathrm{br}} \sqrt{\frac{5}{6}} p_{0} c_{0} \boldsymbol{B}\right]=0, \\
& \frac{\partial}{\partial t} \overline{\mathbf{p}}+2 p_{0}\left(\overline{\boldsymbol{\nabla} \boldsymbol{v}}+\frac{2}{5} p_{0}{ }^{-1} \overline{\boldsymbol{\nabla}}_{\text {trans }}\right) \quad+\omega_{\eta} \overline{\mathbf{p}} \quad+\omega_{\eta \mathrm{T}} p_{0} \sqrt{2} \mathbf{a}=0, \\
& \frac{\partial}{\partial t} \mathbf{a}+\frac{c_{0}}{\sqrt{3}} \boldsymbol{\nabla} \cdot \underline{\mathbf{b}}+\omega_{\mathrm{H}} \mathscr{H}: \mathbf{a} \quad+\omega_{\eta \mathrm{T}} \overline{\mathbf{p}}\left(\sqrt{2} p_{0}\right)^{-1}+\omega_{\mathrm{T}} \mathbf{a}=0, \\
& \frac{\partial}{\partial t} b_{\lambda, \mu v}+\frac{c_{0}}{\sqrt{3}} \frac{\partial}{\partial x_{\lambda}} a_{\mu \nu}+\omega_{\mathrm{H}} \mathscr{H}_{\mu v, \mu^{\prime} v^{\prime}} b_{\lambda, \mu^{\prime} v^{\prime}}+\frac{\sqrt{\frac{6}{5}}}{p_{0} c_{0}} \Delta_{\mu v, \lambda \lambda^{\prime}}\left(\omega_{\mathrm{bt}} \boldsymbol{q}_{\text {trans }}+\omega_{\mathrm{br}} \boldsymbol{q}_{\mathrm{rot}}\right)_{\lambda^{\prime}}+\omega_{\mathrm{b}} b_{\lambda, \mu v}=0 .
\end{aligned}
$$

Three of these equations express the conservation of particles, of energy and of momentum. They can be derived from the nonlinearized Waldmann-Snider equation in their most general form ${ }^{1}$, here the linearized version is given. In (1.3) the linearized continuity equation is stated.

The variables $T_{\text {trans }}, T_{\text {rot }}, \boldsymbol{q}_{\text {trans }}$ and $\boldsymbol{q}_{\text {rot }}$ can be replaced by the temperature $T$, the difference temperature $T^{\prime}$, the total heat flux $\boldsymbol{q}$ and by $\boldsymbol{q}^{\prime}$ :

$$
\begin{array}{ll}
T=c_{\mathrm{t}} T_{\text {trans }}+c_{\mathrm{r}} T_{\text {rot }}, & T^{\prime}=T_{\text {trans }}-T_{\text {rot }}, \\
\boldsymbol{q}=\boldsymbol{q}_{\text {trans }}+\boldsymbol{q}_{\text {rot }}, & \boldsymbol{q}^{\prime}=c_{\mathrm{r}} \boldsymbol{q}_{\text {trans }}-c_{\mathrm{t}} \boldsymbol{q}_{\text {rot }} .
\end{array}
$$

By $c_{\mathrm{t}}$ and $c_{\mathrm{r}}$ the relative translational and rotational heat capacities (at temperature $T_{0}$ ) are denoted

$$
c_{\mathrm{t}}=\frac{\frac{3}{2} k}{\left(\frac{3}{2} k+c_{\mathrm{rot}}^{(0)}\right)}, \quad c_{\mathrm{r}}=\frac{c_{\mathrm{rot}}^{(0)}}{\left(\frac{3}{2} k+c_{\mathrm{rot}}^{(0)}\right)} .
$$

Then (1.4), (1.5) can be transformed into equations for $T$ and $T^{\prime}$. One of them is the linearized form of the energy conservation equation,

$$
n_{0}\left(\frac{3}{2} k+c_{\mathrm{rot}}^{(0)}\right) \partial T / \partial t+\boldsymbol{\nabla} \cdot\left(\boldsymbol{q}+p_{0} \boldsymbol{v}\right)=0,
$$


the second describes the relaxation of $T^{\prime}$

$\partial T^{\prime} / \partial t+\frac{3}{2} T_{0} \boldsymbol{\nabla} \cdot\left[1 /\left(p_{0} c_{\mathrm{r}}\right) \boldsymbol{q}^{\prime}+\boldsymbol{v}\right]+\omega_{\mathrm{T}^{\prime}} T^{\prime}=0$,

$p_{0}=n_{0} k T_{0}$ is the equilibrium pressure. Hence,

$$
\omega_{\mathrm{T}^{\prime}}=\left(1 / c_{\mathrm{r}}\right) \omega_{0}>0
$$

is the relaxation constant of the difference temperature. Via the relation $18,21,22$

$$
\omega_{0}=-{ }_{5}^{6}\left(c_{\mathrm{r}} / c_{\mathrm{t}}\right) \omega_{\mathrm{tr}}
$$

it can be expressed by the coupling coefficient $\omega_{\text {tr }}$ for the heat fluxes.

The linearized momentum equation can in general be written as

$$
n_{0} \boldsymbol{M}(\partial \boldsymbol{v} / \partial t)+\boldsymbol{\nabla} \cdot \mathbf{p}=\mathbf{0},
$$

and the pressure tensor is split up into its irreducible components

$$
\mathbf{p}=\left(p+p^{\prime}\right) \boldsymbol{\delta}+\overline{\mathbf{p}} .
$$

The gas pressure $p$ is introduced by $p=n k T \approx$ $n k T_{0}+n_{0} k T$. Then the comparison with (1.6) shows that the scalar part $p^{\prime}$ of the friction pressure tensor $p^{\prime} \boldsymbol{\delta}+\overline{\mathbf{p}}$ is proportional to the difference temperature $T^{\prime \prime}$ :

$$
p^{\prime}=c_{\mathrm{r}}\left(p_{0} / T_{0}\right) T^{\prime} .
$$

The conservation equations (1.3), (1.6), (1.14) contain no relaxation terms. In contradistinction, relaxation phenomena are described by Eqs. (1.7) (1.11) and (1.15). The relaxation constants $\omega .$. are matrix elements of the linearized Waldmann-Snider collision term, for details see Refs. ${ }^{4,19,20}$. To compare with the notation of Hess ${ }^{4}$ it is noted that

$$
\begin{aligned}
& \frac{3}{5} \frac{c_{\mathrm{r}}}{c_{\mathrm{t}}} \omega_{\mathrm{r}}=\omega_{\mathrm{i}}, \sqrt{\frac{3}{5}} \frac{c_{\mathrm{r}}}{c_{\mathrm{t}}} \omega_{\mathrm{tr}}=\omega_{\mathrm{ti}} \\
& \omega_{\mathrm{b}}=\omega_{\mathrm{k}}, \sqrt{\frac{5}{3}} \omega_{\mathrm{bt}}=\omega_{\mathrm{kt}}, \sqrt{\frac{c_{\mathrm{r}}}{c_{\mathrm{t}}}} \omega_{\mathrm{br}}=\omega_{\mathrm{ki}}
\end{aligned}
$$

Ten relaxation constants occur in Eqs. (1.6) - (1.11), but they are not independent since three exact relations exist. Besides (1.17) these are ${ }^{18,22}$

$$
\omega_{\mathrm{t}}=\frac{2}{3} \omega_{\eta}+{ }_{6}^{5} \omega_{0}
$$

and $18,19,22$

$$
\omega_{\mathrm{bt}}=(1 / \sqrt{5}) \omega_{\eta \mathrm{T}} .
$$

Hence there are seven independent relaxation constants which can be determined from experimental data on shear viscosity, bulk viscosity, thermal conductivity and on the Senftleben-Beenakker effects of viscosity and of thermal conductivity. Numbers will be given in $\S 4$ for the gases HD, $\mathrm{CH}_{4}, \mathrm{CO}$ and $\mathrm{N}_{2}$.

A magnetic field acts directly on the tensor polarization a and its flux $\underline{\mathbf{b}}$ via the magnetic moment associated with the rotational angular momentum $\boldsymbol{J}$ of a molecule. Through the mutual coupling of a with the friction pressure tensor $\overline{\mathbf{p}}$ and of the "Kagan vector" $B_{\mu}=b_{v, v \mu}$ with the heat fluxes, respectively, the flow and heat conduction in the gas are influenced by a magnetic field. The field strength $H$ enters via the precession frequency

$$
\omega_{\mathrm{H}}=(1 / \hbar) g_{\mathrm{r}} \mu_{\mathrm{N}} H
$$

of a molecule with the rotational $g$-factor $g_{\mathrm{r}}, \mu_{\mathrm{N}}$ is the nuclear magneton. The dependence on the field direction $\boldsymbol{h}$ is contained in the second rank tensor $H_{\mu \mu^{\prime}}=\varepsilon_{\mu \lambda \mu^{\prime}} h_{\lambda}$ and in the fourth rank tensor $\mathscr{H}_{\mu v, \mu^{\prime} v^{\prime}}=H_{\mu \mu^{\prime}} \delta_{v v^{\prime}}+H_{v v^{\prime}} \delta_{\mu \mu^{\prime}}$. They describe the infinitesimal rotation of a vector and of a second rank tensor, respectively, around $\boldsymbol{h}^{23}$.

The isotropic tensor

$$
\Delta_{\mu v, \mu^{\prime} v^{\prime}}=\frac{1}{2}\left(\delta_{\mu \mu^{\prime}} \delta_{v v^{\prime}}+\delta_{\mu v^{\prime}} \delta_{v \mu^{\prime}}\right)-\frac{1}{3} \delta_{\mu v} \delta_{\mu^{\prime} v^{\prime}}
$$

projects any second rank tensor on its symmetric traceless component.

The next two paragraphs deal with the derivation of constitutive laws and differential equations from transport relaxation equations.

\section{$\S 2$ Constitutive laws}

If the relaxation processes are fast compared with a macroscopic time scale the time derivatives can be dropped in Eqs. (1.15) and (1.7)-(1.11). Then from (1.15) the difference temperature is expressed by

$$
T^{\prime}=-\frac{1}{\omega_{\mathrm{T}^{\prime}}} \frac{2}{3} T_{0} \boldsymbol{\nabla} \cdot\left(\boldsymbol{v}+\frac{1}{p_{0} c_{\mathrm{r}}} \boldsymbol{q}^{\prime}\right) .
$$

By the use of (1.18) an ansatz for the scalar component $p^{\prime}$ of the friction pressure tensor is obtained

$$
p^{\prime}=-\eta_{\mathbf{v}} \boldsymbol{\nabla} \cdot\left(\boldsymbol{v}+\frac{1}{p_{0} c_{\mathrm{r}}} \boldsymbol{q}^{\prime}\right) .
$$

Hence the bulk viscosity $\eta_{\mathrm{v}}$ is related to the relaxation constant of $T^{\prime \prime}$,

$$
\eta_{\mathrm{V}}=\frac{2}{3} c_{\mathrm{r}}\left(p_{0} / \omega_{\mathrm{T}^{\prime}}\right)=\frac{2}{3} c_{\mathrm{r}}^{2}\left(p_{0} / \omega_{0}\right),
$$

it vanishes for a monatomic dilute gas $\left(c_{\mathrm{r}}=0\right)^{22,24}$. According to (1.9) the symmetric traceless compo- 
nent $\overline{\mathbf{p}}$ of the friction pressure tensor is given by $\overline{\mathbf{p}}=-2 \eta_{\text {iso }}\left[\overline{\boldsymbol{\nabla} \boldsymbol{v}}+\frac{2}{5} p_{0}^{-1} \overline{\boldsymbol{\nabla}} \boldsymbol{q}_{\text {trans }}\right]$

where

$$
-p_{0} \sqrt{2}\left(\omega_{\eta \mathrm{T}} / \omega_{\eta}\right) \mathbf{a}
$$

$$
\eta_{\text {iso }}=p_{0} / \omega_{\eta}
$$

is the isotropic shear viscosity. The adjective "isotropic" is used whenever the anisotropic alignments $\mathbf{a}$ and $\underline{\mathbf{b}}$ are neglected.

A magnetic field influences the flow of a polyatomic gas through the coupling of $\overline{\mathbf{p}}$ with the tensor polarization a. F or the heat conduction, the coupling of the heat fluxes with the Kagan vector is of importance, cf. Eqs. (1.7), (1.8):

$$
\begin{aligned}
& \boldsymbol{q}_{\text {trans }}=-\lambda_{\text {iso }}^{\mathrm{t}}\left(\boldsymbol{\nabla} T_{\text {trans }}+\frac{2}{5} \frac{T_{0}}{p_{0}} \boldsymbol{\nabla} \cdot \overline{\mathbf{p}}\right)-\lambda_{\text {iso }}^{\mathrm{tr}} \boldsymbol{\nabla} T_{\text {rot }}-\frac{p_{0} c_{0}}{V^{2}} a_{\mathrm{bt}} / \omega_{\mathrm{b}} / \omega_{\mathrm{t}}\left(1-A_{\mathrm{tr}}\right)^{-1 / 2} \boldsymbol{B}, \\
& \boldsymbol{q}_{\text {rot }}=-\lambda_{\text {iso }}^{\mathrm{rt}}\left(\boldsymbol{\nabla} T_{\text {trans }}+\frac{2}{5} \frac{T_{0}}{p_{0}} \boldsymbol{\nabla} \cdot \overline{\mathbf{p}}\right)-\lambda_{\text {iso }}^{\mathrm{r}} \boldsymbol{\nabla} T_{\text {rot }}-\frac{p_{0} c_{0}}{\sqrt{2}} a_{\mathrm{br}} \sqrt{\omega_{\mathrm{b}} / \omega_{\mathrm{r}}}\left(1-A_{\mathrm{tr}}\right)^{-1 / 2} \boldsymbol{B} .
\end{aligned}
$$

The matrix of the isotropic heat conductivities

$$
\begin{aligned}
& \lambda_{\mathrm{iso}}^{\mathrm{t}}=\frac{5}{2} \frac{k}{M} \frac{p_{0}}{\omega_{\mathrm{t}}}\left(1-A_{\mathrm{tr}}\right)^{-1}, \quad \lambda_{\mathrm{iso}}^{\mathrm{r}}=\frac{5}{2} \frac{k}{M} \frac{p_{0}}{\omega_{\mathrm{r}}}\left(1-A_{\mathrm{tr}}\right)^{-1}, \\
& \lambda_{\mathrm{iso}}^{\mathrm{tr}}=\lambda_{\text {iso }}^{\mathrm{rt}}=\frac{5}{2} \frac{k}{M} \frac{\left(-\omega_{\mathrm{tr}}\right) p_{0}}{\omega_{\mathrm{t}} \omega_{\mathrm{r}}}\left(1-A_{\mathrm{tr}}\right)^{-1}
\end{aligned}
$$

is positive definite,

$$
\lambda_{\text {iso }}^{\mathrm{t}}, \lambda_{\text {iso }}^{\mathrm{r}}, \lambda_{\text {iso }}^{\mathrm{tr}}>0,\left(\lambda_{\mathrm{t}}^{\mathrm{t}} \lambda^{\mathrm{r}}-\left(\lambda^{\mathrm{tr}}\right)^{2}\right)_{\text {iso }}>0,
$$

since this is true for the relevant relaxation constants ${ }^{19}$

$$
\omega_{\mathrm{t}}, \omega_{\mathrm{r}},-\omega_{\mathrm{tr}}>0,1-A_{\mathrm{tr}}>0, A_{\mathrm{tr}} \equiv \omega_{\mathrm{tr}}^{2} /\left(\omega_{\mathrm{t}} \omega_{\mathrm{r}}\right) .
$$

In Eq. (2.4) the second contribution to the friction pressure tensor $\overline{\mathbf{p}}\left[\sim \frac{2}{5} p_{0}{ }^{-1} \overline{\boldsymbol{\nabla}} \boldsymbol{q}_{\text {trans }}\right]$ represents thermal stresses which become important at lower mean pressures $p_{0}$. For the translational heat flux the ansatz (2.6) has to be used, it is coupled to the pressure tensor by the term $\sim \frac{2}{5} T_{0} p_{0}^{-1} \nabla \cdot \overrightarrow{\mathbf{p}}$. In a steady state $\boldsymbol{\nabla} \cdot \overline{\mathbf{p}}$ can be replaced by [see (3.7)] $-\boldsymbol{\nabla} p-c_{\mathrm{r}} p_{0} T_{0}{ }^{-1} \boldsymbol{\nabla} T^{\prime}$. If a Chapman-Enskog procedure is used 20 instead of the moment method these "Burnett terms" are given by higher order derivatives of temperature $T$ (instead of $\overline{\boldsymbol{\nabla}}_{\text {trans }}$ ) and of velocity (instead of $\boldsymbol{\nabla} \cdot \overline{\mathbf{p}}$ ), respectively.

The strength of the coupling of the Kagan vector $\boldsymbol{B}$ with the translational and rotational heat fluxes is determined by the quantities $a_{\mathrm{bt}}$ and $a_{\mathrm{br}}$, respectively:

$$
\begin{aligned}
& a_{\mathrm{bt}}=\sqrt{\frac{5}{3}}\left[\frac{\omega_{\mathrm{bt}}}{\sqrt{\omega_{\mathrm{b}} \omega_{\mathrm{t}}}}+\frac{\omega_{\mathrm{br}}}{\sqrt{\omega_{\mathrm{b}} \omega_{\mathrm{r}}}} \frac{\left(-\omega_{\mathrm{tr}}\right)}{\sqrt{\omega_{\mathrm{t}} \omega_{\mathrm{r}}}}\right]\left(1-A_{\mathrm{tr}}\right)^{-1 / 2},
\end{aligned}
$$

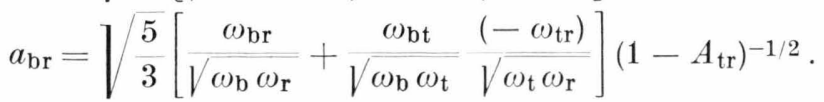

The corresponding equations for the heat fluxes $\boldsymbol{q}$ and $\boldsymbol{q}^{\prime}$ are given by

$$
\begin{gathered}
\boldsymbol{q}=-\lambda_{\mathrm{iso}} \boldsymbol{\nabla} T-\lambda_{\mathrm{iso}}^{\prime \prime} \boldsymbol{\nabla} T^{\prime}-\frac{2}{5} \frac{T_{0}}{p_{0}}\left(\lambda_{\mathrm{iso}}^{\mathrm{t}}+\lambda_{\mathrm{iso}}^{\mathrm{tr}}\right) \boldsymbol{\nabla} \cdot \overline{\mathbf{p}}-\frac{p_{0} c_{0}}{\sqrt{2}} a_{\mathrm{rbt}} \sqrt{\frac{\omega_{\mathrm{b}}}{\omega_{\mathrm{t}}} \frac{\lambda_{\text {iso }}}{\lambda_{\text {iso }}^{\mathrm{t}}}\left(1-A_{\mathrm{tr}}\right)^{-1 / 2} \boldsymbol{B},} \\
\boldsymbol{q}^{\prime}=-\lambda_{\text {iso }}^{\prime \prime} \boldsymbol{\nabla} T-\lambda_{\text {iso }}^{\prime} \boldsymbol{\nabla} T^{\prime}-\frac{2}{5} \frac{T_{0}}{p_{0}}\left(c_{\mathrm{r}} \lambda_{\mathrm{iso}}^{\mathrm{t}}-c_{\mathrm{t}} \lambda_{\mathrm{iso}}^{\mathrm{r}}\right) \boldsymbol{\nabla} \cdot \overline{\mathbf{p}}-\frac{p_{0} c_{0}}{\sqrt{2}} a_{\mathrm{rbt}}^{\prime} \sqrt{\frac{\omega_{\mathrm{b}}}{\omega_{\mathrm{t}}} \frac{\lambda_{\text {iso }}^{\prime}}{\lambda_{\text {iso }}^{\mathrm{t}}}\left(1-A_{\mathrm{tr}}\right)^{-1 / 2} \boldsymbol{B} .}
\end{gathered}
$$

The isotropic heat conductivity coefficients $\lambda_{\text {iso }}, \lambda_{\text {iso }}^{\prime}, \lambda_{\text {iso }}^{\prime \prime}$ are linear combinations of $\lambda_{\text {iso }}^{\mathrm{t}}, \lambda_{\text {iso }}^{\mathrm{r}}, \lambda_{\text {iso }}^{\mathrm{tr}}$ :

$$
\lambda_{\text {iso }}=\lambda_{\text {iso }}^{\mathrm{t}}+\lambda_{\text {iso }}^{\mathrm{r}}+2 \lambda_{\text {iso }}^{\mathrm{tr}}, \lambda_{\text {iso }}^{\prime}=c_{\mathrm{r}}^{2} \lambda_{\text {iso }}^{\mathrm{t}}+c_{\mathrm{t}}^{2} \lambda_{\text {iso }}^{\mathrm{r}}-2 c_{\mathrm{r}} c_{\mathrm{t}} \lambda_{\text {iso }}^{\mathrm{tr}}, \lambda_{\text {iso }}^{\prime \prime}=c_{\mathrm{r}} \lambda_{\text {iso }}^{\mathrm{t}}-c_{\mathrm{t}} \lambda_{\text {iso }}^{\mathrm{r}}+\left(c_{\mathrm{r}}-c_{\mathrm{t}}\right) \lambda_{\mathrm{iso}}^{\mathrm{tr}} .
$$


From (2.9) the following inequalities are derived:

$$
\lambda_{\text {iso }}>0, \lambda_{\text {iso }}^{\prime}>0, \lambda_{\text {iso }} \lambda_{\text {iso }}^{\prime}-\left(\lambda_{\text {iso }}^{\prime \prime}\right)^{2}>0 .
$$

These expressions for the isotropic transport constants $\eta_{\text {iso }}, \lambda_{\text {iso }}, \lambda_{\text {iso }}^{\prime}$ and $\lambda_{\text {iso }}^{\prime \prime}$ are obtained from the Waldmann-Snider equation by neglect of the tensor polarization and its flux, hence they coincide with the results which follow from the Wang Chang-Uhlenbeck equation ${ }^{22,} 24$.

The quantities $a_{\mathrm{rbt}}$ and $a_{\mathrm{rbt}}^{\prime}$ characterize the coupling of the heat fluxes $\boldsymbol{q}$ and $\boldsymbol{q}^{\prime}$ with the Kagan vector $\boldsymbol{B}$ :

$$
a_{\mathrm{rbt}}=\sqrt{\lambda_{\mathrm{iso}}^{\mathrm{t}} / \lambda_{\mathrm{iso}}}\left(a_{\mathrm{bt}}+\sqrt{\omega_{\mathrm{t}} / \omega_{\mathrm{r}}} a_{\mathrm{br}}\right), \quad a_{\mathrm{rbt}}^{\prime}=\sqrt{\lambda_{\mathrm{iso}}^{\mathrm{t}} / \lambda_{\mathrm{iso}}^{\prime}}\left(c_{\mathrm{r}} a_{\mathrm{bt}}-c_{\mathrm{t}} \sqrt{\omega_{\mathrm{t}} / \omega_{\mathrm{r}}} a_{\mathrm{br}}\right) .
$$

An evaluation of the magnetic field dependent Eqs. (1.10) and (1.11) is most easily performed by use of the fourth rank projection tensors $\mathscr{P}_{\mu \nu, \mu^{\prime} \nu^{\prime}}^{(m)}(m=0, \pm 1, \pm 2)$ introduced by Hess ${ }^{3},{ }^{23}$. Due to the property

the $m$-component

$$
\mathscr{H}_{\mu v, \mu^{\prime \prime} v^{\prime \prime}} \mathscr{P}_{\mu^{\prime \prime} \nu^{\prime \prime}, \mu^{\prime} v^{\prime}}^{(m)}=i m \mathscr{P}_{\mu \nu, \mu^{\prime} v^{\prime}}^{(m)}
$$

$$
b_{\lambda, \mu \nu}^{(m)} \equiv \mathscr{P}_{\mu \nu, \mu^{\prime} v^{\prime}}^{(m)} b_{\lambda, \mu^{\prime} v^{\prime}}
$$

of the tensor polarization flux can be easily computed. Since the projection tensors obey the completeness relation

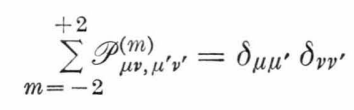

the tensor polarization flux is obtained by summation over its $m$-components:

$$
b_{\lambda, \mu \nu}=\sum_{m=-2}^{+2} \frac{1}{1+i m \varphi_{\mathrm{b}}} \mathscr{P}_{\mu \nu, \mu^{\prime} v^{\prime}}^{(m)}\left(-l_{\mathrm{b}} \frac{\partial a_{\mu^{\prime} v^{\prime}}}{\partial x_{\lambda}}+\Delta_{\mu^{\prime} v^{\prime}, \lambda^{\prime} \lambda} a_{\lambda^{\prime}}^{\mathrm{rt}}\right),
$$

$l_{\mathrm{b}}=c_{0} /\left(\sqrt{3} \omega_{\mathrm{b}}\right)$ is a mean free path, $\varphi_{\mathrm{b}}=\omega_{\mathrm{H}} / \omega_{\mathrm{b}}$ is a precession angle. According to (1.11) the vector $\boldsymbol{a}^{\mathrm{rt}}$ is a linear combination of $\boldsymbol{q}_{\text {trans }}$ and $\boldsymbol{q}_{\text {rot }}$. Since the field effects are relatively small ${ }^{2}$ the isotropic approximation can be used for the heat fluxes (i.e. $\boldsymbol{B}$ is neglected) in order to calculate the tensor polarization flux. With the aid of Eqs. (2.6), (2.7) and (2.10), (2.15) the following expression is found:

$$
\boldsymbol{a}^{\mathrm{rt}}=\frac{3}{5} \frac{\sqrt{\mathbf{2}}}{p_{0} c_{0}} \lambda_{\text {iso }} \sqrt{\frac{\lambda_{\text {iso }}^{\mathrm{t}}}{\lambda_{\text {iso }}} \frac{\omega_{\mathrm{t}}}{\omega_{\mathrm{b}}}\left(1-A_{\mathrm{tr}}\right)}\left(a_{\mathrm{rbt}} \boldsymbol{\nabla} T+a_{\mathrm{rbt}} \sqrt{\frac{\lambda_{\text {iso }}^{\prime}}{\lambda_{\text {iso }}}} \boldsymbol{\nabla} T^{\prime}+\frac{2}{5} \frac{T_{0}}{p_{0}} a_{\mathrm{bt}} V \lambda_{\text {iso }}^{\mathrm{t}} / \lambda_{\text {iso }} \boldsymbol{\nabla} \cdot \overline{\mathbf{p}}\right) .
$$

By contraction an ansatz for the Kagan vector $B_{\mu}=b_{v, v \mu}$ is derived from (2.16)

The second rank tensor

$$
\boldsymbol{B}={ }_{3}^{5}\left(\boldsymbol{\delta}-\boldsymbol{\Lambda}\left(\varphi_{\mathrm{b}}\right)\right) \cdot \boldsymbol{a}^{\mathrm{rt}}-l_{\mathrm{b}} \boldsymbol{\nabla} \cdot\left(\boldsymbol{\Delta}-\boldsymbol{\Pi}\left(\varphi_{\mathrm{b}}\right)\right): \mathbf{a} .
$$

$$
\boldsymbol{\Lambda}\left(\varphi_{\mathrm{b}}\right)=\frac{3}{5} \frac{\varphi_{\mathrm{b}}{ }^{2}}{1+\varphi_{\mathrm{b}}{ }^{2}} \mathbf{P}^{\|}+\frac{3}{10}\left(\frac{\varphi_{\mathrm{b}}{ }^{2}}{1+\varphi_{\mathrm{b}}{ }^{2}}+\frac{8 \varphi_{\mathrm{b}}{ }^{2}}{1+4 \varphi_{\mathrm{b}}{ }^{2}}\right) \mathbf{P}^{\perp}+\frac{3}{10}\left(\frac{\varphi_{\mathrm{b}}}{1+\varphi_{\mathrm{b}}{ }^{2}}+\frac{4 \varphi_{\mathrm{b}}}{1+4 \varphi_{\mathrm{b}}{ }^{2}}\right) \mathbf{P}^{\operatorname{tr}},
$$

with

$$
P_{\mu \nu}^{\|}=h_{\mu} h_{\nu}, \quad P_{\mu \nu}^{\perp}=\delta_{\mu \nu}-h_{\mu} h_{\nu}, \quad P_{\mu \nu}^{\mathrm{tr}}=\varepsilon_{\mu \lambda \nu} h_{\lambda},
$$

determines the field dependent heat conductivity tensor in the hydrodynamic limit [see (4.7)], whereas the fourth rank tensor

$$
\boldsymbol{\Pi}\left(\varphi_{\mathrm{b}}\right)=\sum_{m=1}^{2}\left[\frac{m^{2} \varphi_{\mathrm{b}}{ }^{2}}{1+m^{2} \varphi_{\mathrm{b}}{ }^{2}}(\mathscr{P}(m)+\mathscr{P}(-m))+\frac{m \varphi_{\mathrm{b}}}{1+m^{2} \varphi_{\mathrm{b}}{ }^{2}} i(\mathscr{P}(m)-\mathscr{P}(-m))\right]
$$

will give the viscosity tensor [see (4.2)].

The constitutive laws (2.2), (2.4), (2.11), (2.12) and (2.16) for $p^{\prime}, \overline{\mathbf{p}}, \boldsymbol{q}, \boldsymbol{q}^{\prime}$ and $\underline{\mathbf{b}}$ contain the temperatures $T$ and $T^{\prime}$, the mean velocity $\boldsymbol{v}$ and the tensor polarization a. In the next paragraph differential equations for these quantities will be derived. 


\section{$\S 3$ Differential equations}

According to (1.10) the $m$-component of the tensor polarization is given by

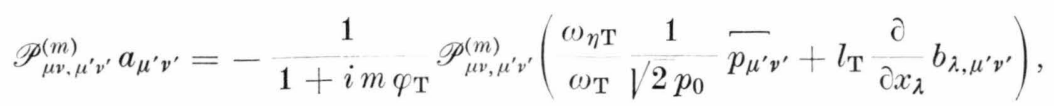

$\varphi_{\mathrm{T}}=\omega_{\mathrm{H}} / \omega_{\mathrm{T}}$ is a precession angle, $l_{\mathrm{T}}=c_{0} /\left(1 / 3 \omega_{\mathrm{T}}\right)$ is a mean free path. If the coupling of $\overrightarrow{\mathbf{p}}$ with a is neglected in the ansatz (2.4) and if the expression (2.16) for $\underline{\mathbf{b}}$ is used together with 23

$$
\mathscr{P}_{\mu v, \mu^{\prime \prime} v^{\prime \prime}}^{(m)} \mathscr{P}_{\mu^{\prime} v^{\prime \prime}, \mu^{\prime} v^{\prime}}^{\left(m^{\prime}\right)} \equiv\left(\mathscr{P}(m): \mathscr{P}\left(m^{\prime}\right)\right)_{\mu v, \mu^{\prime} v^{\prime}}=\delta_{m m^{\prime}} \mathscr{P}_{\mu v, \mu^{\prime} v^{\prime}}^{(m)}
$$

the following differential equation for the $m$-component of the tensor polarization is obtained from (3.1) $\left(1-L^{2}{ }_{m} \boldsymbol{\nabla} \cdot \boldsymbol{\nabla}\right) \mathscr{P ( m )}: \mathbf{a}$

$$
=\frac{1}{1+i m \varphi_{\mathrm{T}}} \mathscr{P}(m):\left[\frac{\sqrt{\mathbf{2} \omega_{\eta \mathrm{T}}}}{\omega_{\eta} \omega_{\mathrm{T}}}\left(\overline{\boldsymbol{\nabla} \boldsymbol{v}}+\frac{2}{5} p_{0}^{-1} \overline{\boldsymbol{\nabla}}_{\text {trans }}\right)-\frac{l_{\mathrm{T}}}{1+i m \varphi_{\mathrm{b}}} \overline{\boldsymbol{\nabla} \boldsymbol{a}^{\mathrm{rt}}}\right], m=0, \pm 1, \pm 2 .
$$

The vector $\boldsymbol{a}^{\text {rt }}$ is stated in (2.17), for the translational heat flux the isotropic approximation can be used, i.e. $\boldsymbol{B}$ can be neglected in (2.6). The abbreviation $L_{m}$ denotes a field dependent mean free path,

$$
L_{m}=\sqrt{l_{\mathrm{b}} l_{\mathrm{T}} /\left[\left(1+i m \varphi_{\mathrm{b}}\right)\left(1+i m \varphi_{\mathrm{T}}\right)\right]}, \quad m=0, \pm 1, \pm 2 .
$$

The differential equations (3.2) apply to steady states and to processes with a time constant that is large compared to the relaxation time $\omega_{\mathrm{T}}^{-1}$. Now, differential equations for $T, T^{\prime}$ and $\boldsymbol{v}$ will be derived for the steady state only.

Then the continuity equation (1.3) reduces to

$$
\boldsymbol{\nabla} \cdot \boldsymbol{v}=0
$$

and consequently the conservation equation (1.14) for the energy gives

$$
\boldsymbol{\nabla} \cdot \boldsymbol{q}=0 .
$$

From Eq. (2.1) the difference temperature is expressed by

$$
T^{\prime}=-\frac{2}{3} \frac{T_{0}}{p_{0}} \frac{1}{\omega_{0}} \boldsymbol{\nabla} \cdot \boldsymbol{q}^{\prime}=-\frac{2}{3} \frac{T_{0}}{p_{0}} \frac{1}{\omega_{0}} \boldsymbol{\nabla} \cdot \boldsymbol{q}_{\text {trans }} .
$$

In a steady state the linearized momentum equation (1.6) reduces to

$$
\boldsymbol{\nabla} \cdot \overline{\mathbf{p}}+\boldsymbol{\nabla} p+c_{\mathrm{r}}\left(p_{0} / T_{0}\right) \boldsymbol{\nabla} T^{\prime}=\mathbf{0} .
$$

This condition is evaluated by insertion of the ansatz (2.4) for $\overline{\mathrm{p}}$ and by the use of Eqs. (3.4), (3.5), (3.6) with the result

$$
{ }_{\eta_{\text {iso }}}^{1} \boldsymbol{\nabla} p-\boldsymbol{\nabla} \cdot \boldsymbol{\nabla} \boldsymbol{v}=\frac{2}{5} p_{0}^{-1} \boldsymbol{\nabla} \cdot \boldsymbol{\nabla} \boldsymbol{q}_{\text {trans }}-{ }_{\eta_{\text {iso }}}^{1} \frac{p_{0}}{T_{0}}\left(c_{\mathrm{r}}+\frac{1}{5} \frac{\omega_{0}}{\omega_{\eta}}\right) \boldsymbol{\nabla} T^{\prime}+\sqrt{2} \omega_{\eta \mathrm{T}} \boldsymbol{\nabla} \cdot \mathbf{a} .
$$

By differentiation of (3.8) a second order differential equation for the pressure is derived

$$
\boldsymbol{\nabla} \cdot \boldsymbol{\nabla} p=-\frac{p_{0}}{T_{0}}\left(c_{\mathrm{r}}+\frac{4}{5} \frac{\omega_{0}}{\omega_{\eta}}\right) \boldsymbol{\nabla} \cdot \boldsymbol{\nabla} T^{\prime}+p_{0} \sqrt{\mathbf{2}} \frac{\omega_{\eta \mathrm{T}}}{\omega_{\eta}} \boldsymbol{\nabla} \cdot(\boldsymbol{\nabla} \cdot \mathbf{a})
$$

Now, Eq. (3.5) can be evaluated if the ansatz (2.11) for the heat flux $\boldsymbol{q}$ is introduced and if Eqs. (3.7), (3.9) are used. The result is a modified Fourier equation for the temperature $T$ :

$$
\begin{aligned}
\boldsymbol{\nabla} \cdot \boldsymbol{\nabla} T=-\boldsymbol{\nabla} \cdot \boldsymbol{\nabla} T^{\prime}\left(\frac{\lambda_{\text {iso }}^{\prime \prime}}{\lambda_{\text {iso }}}\right. & \left.+\frac{8}{25} \frac{\lambda_{\text {iso }}^{\text {trans }}}{\lambda_{\text {iso }}} \frac{\omega_{0}}{\omega_{\eta}}\right)+\frac{2}{5} T_{0} \frac{\lambda_{\text {iso }}^{\text {trans }}}{\lambda_{\text {iso }}} \sqrt{2} \frac{\omega_{\eta \mathrm{T}}}{\omega_{\eta}} \boldsymbol{\nabla} \cdot(\boldsymbol{\nabla} \cdot \mathbf{a}) \\
& -\frac{p_{0} c_{0}}{\sqrt{ } \mathbf{2}}\left(\frac{\omega_{\mathrm{t}}}{\omega_{\mathrm{b}}}\left(1-A_{\mathrm{tr}}\right) \lambda_{\text {iso }} \lambda_{\text {iso }}^{\mathrm{t}}\right)^{-1 / 2} a_{\mathrm{rbt}} \boldsymbol{\nabla} \cdot \boldsymbol{B} .
\end{aligned}
$$


The modifications are due to the polyatomic nature of the gas, i.e. due to derivatives of the difference temperature $T^{\prime}$ and of the polarizations a and $\underline{\mathbf{b}}$.

The isotropic translational heat conductivity is denoted by

$$
\lambda_{\text {iso }}^{\text {trans }}=\lambda_{\text {iso }}^{\mathrm{t}}+\lambda_{\text {iso }}^{\text {tr }} \text {. }
$$

A differential equation for $T^{\prime}$ is derived from (3.6) by use of the ansatz (2.12) for $\boldsymbol{q}^{\prime}$ and of Eqs. (3.7), (3.9), (3.10):

$$
T^{\prime}-L_{\mathrm{T}^{\prime}}^{2} \boldsymbol{\nabla} \cdot \boldsymbol{\nabla} T^{\prime}=\frac{T_{0} L_{\mathrm{T}^{\prime}}^{2}}{1+\frac{8}{25} \frac{\omega_{0}}{\omega_{\eta}}}\left[\frac{2}{5} V^{2} \frac{\omega_{\eta \mathrm{T}}}{\omega_{\eta}} \boldsymbol{\nabla} \cdot(\boldsymbol{\nabla} \cdot \mathbf{a})-\sqrt{\frac{2}{5} \frac{1}{l_{\mathrm{b}}}}\left(\frac{\omega_{\mathrm{bt}}}{\omega_{\mathrm{b}}}-\frac{\omega_{\mathrm{br}}}{\omega_{\mathrm{b}}}\right) \boldsymbol{\nabla} \cdot \boldsymbol{B}\right] .
$$

The spatial variation of $T^{\prime}$ is characterized by the mean free path $L_{\mathrm{T}^{\prime}}$ given by

$$
L_{\mathrm{T}^{\prime}}^{2}=\frac{5}{3} \frac{k T_{0}}{M} \frac{1}{\omega_{\mathrm{r}} \omega_{0}} \frac{\lambda_{\text {iso }}^{\mathrm{t}}}{\lambda_{\text {iso }}}\left(1+\frac{8}{25} \omega_{\eta}\right) .
$$

Finally, the term $\boldsymbol{\nabla} \cdot \boldsymbol{\nabla} \boldsymbol{q}_{\text {trans }}=\boldsymbol{\nabla} \cdot \boldsymbol{\nabla}\left(c_{\mathrm{t}} \boldsymbol{q}+\boldsymbol{q}^{\prime}\right)$ can be eliminated from Eq. (3.8) by use of the constitutive laws (2.11), (2.12) and of the differential Eqs. (3.10), (3.12). Then a modified Navier-Stokes equation for the mean velocity is obtained, with modifications due to the polyatomic nature of the gas:

$$
\begin{aligned}
1 / \eta_{\text {iso }} \boldsymbol{\nabla} p-\boldsymbol{\nabla} \cdot \boldsymbol{\nabla} \boldsymbol{v}=-\left(c_{\mathrm{r}} \omega_{\eta}+\right. & \left.\frac{4}{5} \omega_{0}\right) T_{0}^{-1} \boldsymbol{\nabla} T^{\prime}+\sqrt{\mathbf{2}} \omega_{\eta \mathrm{T}} \boldsymbol{\nabla} \cdot \mathbf{a} \\
& +\frac{2}{5} c_{0} / \sqrt{\mathbf{2}}\left(\omega_{\mathrm{t}} / \omega_{\mathrm{b}}\left(1-A_{\mathrm{tr}}\right)\right)^{-1 / 2} a_{\mathrm{bt}}(\boldsymbol{\nabla} \boldsymbol{\nabla} \cdot \boldsymbol{B}-\boldsymbol{\nabla} \cdot \boldsymbol{\nabla} \boldsymbol{B}) .
\end{aligned}
$$

The differential Eqs. (3.10), (3.12) for the temperatures $T$ and $T^{\prime},(3.14)$ for the flow velocity $v$ and (3.2) for the tensor polarization a can only be solved if boundary conditions are available for these quantities. In the second part of this paper boundary conditions will be derived. But before this is done some remarks are made on the hydrodynamic limit for differential equations and constitutive laws. Furthermore, numbers for the relaxation constants of the gases $\mathrm{HD}, \mathrm{CH}_{4}, \mathrm{CO}$ and $\mathrm{N}_{2}$ are extracted from experimental data.

\section{$\S 4$ Hydrodynamic approximation. Values for relaxation constants}

For a dilute gas the hydrodynamic limit can be used. The term "hydrodynamic" indicates that only the lowest power in $p_{0}^{-1}$ is retained in any constitutive law or differential equation. Especially the velocity $\boldsymbol{v}$ and the temperature $T$ are determined for vanishing mean free path.

According to (2.16), (2.17) the tensor polarization flux $\underline{\mathbf{b}}$ is of the order $p_{0}{ }^{-1}$ and contains $\boldsymbol{\nabla} T$ and $\boldsymbol{\nabla} T^{\prime}$ only, since $\boldsymbol{\nabla}$ a has been neglected. Similarly, the derivative of $\underline{\mathbf{b}}$ is dropped in (3.1) and $\overline{\mathbf{p}}$ is replaced by $\overline{\boldsymbol{\nabla} \boldsymbol{v}}$, i.e. instead of a differential equation an ex- plicit expression for the tensor polarization is obtained. It shows that $\mathbf{a}$ is of the order $p_{0}^{-1}$ and is determined by $\overrightarrow{\boldsymbol{\nabla} \boldsymbol{v}}$ only. An inspection of Eq. (3.12) reveals then that $T^{\prime}$ is at least of the order $p_{0}{ }^{-2}$ (away from walls) and can be neglected compared to $T$ :

$$
T^{\prime}=0 \text {. }
$$

Consequently the quantities a, $\overline{\mathbf{p}}, \boldsymbol{B}$ and $\boldsymbol{q}$ can be expressed by first order derivatives of $\boldsymbol{v}$ and of $T$ respectively. For the friction pressure tensor and the heat flux the following wellknown expressions are obtained 23,25 :

$$
\begin{aligned}
& \overline{\mathbf{p}}=-2 \eta\left[\boldsymbol{\Delta}-A_{\eta \mathrm{T}} \boldsymbol{\Pi}\left(\varphi_{\mathrm{T}}\right)\right]: \overline{\boldsymbol{\nabla} \boldsymbol{v}}=-2 \sum_{m=-2}^{+2} \eta^{(m)} \mathscr{P}(m): \overline{\boldsymbol{\nabla} \boldsymbol{v}}, \\
& \boldsymbol{q}=-\lambda\left[\boldsymbol{\delta}-a_{\mathrm{rbt}}^{2} \boldsymbol{\Lambda}\left(\varphi_{\mathrm{b}}\right)\right] \cdot \boldsymbol{\nabla} T=-\boldsymbol{\lambda} \cdot \boldsymbol{\nabla} T .
\end{aligned}
$$

Second order differential equations for $T$ and $\boldsymbol{v}$ are then derived from (3.5), $\boldsymbol{\nabla} \cdot \boldsymbol{q}=0$, and (3.7), $\boldsymbol{\nabla} p+\boldsymbol{\nabla} \cdot \overline{\mathbf{p}}=\mathbf{0}$. 
Due to the collisional induced polarizations the field free values of shear viscosity

$$
\eta=\eta_{\text {iso }}\left(1+A_{\eta \mathrm{T}}\right), \quad A_{\eta \mathrm{T}}=\omega^{2} \eta \mathrm{T} /\left(\omega_{\eta} \omega_{\mathrm{T}}\right),
$$

and of thermal conductivity

$$
\lambda=\lambda_{\text {iso }}\left(1+a_{\mathrm{rbt}}^{2}\right)
$$

are higher than the isotropic values $\eta_{\text {iso }}, \lambda_{\text {iso }}$. The relative change is of the same order $\left(A_{\eta \mathrm{T}}, a_{\mathrm{rbt}}^{2}\right)$ as the influence of a magnetic field on the flow and heat conduction in the gas.

According to $(2.20)$ the viscosity coefficients introduced by Hess and Waldmann 23

$$
\eta^{(m)}=\eta\left(1+\varepsilon_{m}+i \varepsilon_{m}^{\mathrm{tr}}\right), \quad m=0, \pm 1, \pm 2,
$$

are given by 23

$$
\begin{gathered}
\varepsilon_{m}=-A_{\eta \mathrm{T}} m^{2} \varphi_{\mathrm{T}^{2}} /\left(1+m^{2} \varphi_{\mathrm{T}^{2}}\right), \\
\varepsilon_{m}^{\mathrm{tr}}=-A_{\eta \mathrm{T}} m \varphi_{\mathrm{T}} /\left(1+m^{2} \varphi_{\mathrm{T}^{2}}\right) .
\end{gathered}
$$

Here, $\eta^{(0)}=\eta$ applies due to the fact that only the tensor polarization has been taken into account for the calculation of the pressure tensor $\overline{\mathbf{p}}$.

The independent components $\lambda_{\|}, \lambda_{\perp}$ and $\lambda_{\text {trans } v}$ of the heat conductivity tensor

$$
\lambda=\lambda_{\|} \mathbf{P}^{\|}+\lambda_{\perp} \mathbf{P}^{\perp}+\lambda_{\operatorname{transv}} \mathbf{P}^{\operatorname{tr}}
$$

are obtained from (2.19) as 25 :

$$
\begin{aligned}
& \lambda_{\|}=\lambda\left[1-\frac{3}{5} a_{\mathrm{rbt}}^{2} \frac{\varphi_{\mathrm{b}}^{2}}{1+\varphi_{\mathrm{b}}^{2}}\right], \\
& \lambda_{\perp}=\lambda\left[1-\frac{3}{10} a_{\mathrm{rbt}}^{2}\left(\frac{\varphi_{\mathrm{b}}^{2}}{1+\varphi_{\mathrm{b}}{ }^{2}}+\frac{8 \varphi_{\mathrm{b}}^{2}}{1+4 \varphi_{\mathrm{b}}^{2}}\right)\right], \\
& \lambda_{\operatorname{trans} v}=-\lambda \frac{3}{10} a_{\mathrm{rbt}}^{2}\left(\frac{\varphi_{\mathrm{b}}}{1+\varphi_{\mathrm{b}}{ }^{2}}+\frac{4 \varphi_{\mathrm{b}}}{1+4 \varphi_{\mathrm{b}}{ }^{2}}\right) .
\end{aligned}
$$

From an analysis of experimental data on the Senftleben-Beenakker effect of viscosity with Eqs. (4.6) values of $\left(H / p_{0}\right)_{q_{\mathrm{T}}=1}$ and $A_{\eta \mathrm{T}}$ can be extracted. The evaluation of thermal conductivity data gives for example the high field limit of $\Delta \lambda_{\perp} / \lambda$ and the position $\left(H / p_{0}\right)_{1 / 2}^{\perp}$ where half of this saturation value is reached, this is for $\left(\varphi_{\mathrm{b}}\right)_{1 / 2}^{\perp}=0.6248$. The absolute value of $a_{\text {rbt }}$ can be determined from

$$
\left(-\frac{\Delta \lambda_{\perp}}{\lambda}\right)_{\mathrm{Sat}} \equiv\left(-\frac{\lambda_{\perp}-\lambda}{\lambda}\right)_{\mathrm{sat}}=\frac{9}{10} a_{\mathrm{rbt}}^{2} .
$$

If $H / p_{0}$ is known for a particular $\varphi .$. then the ratio $\omega . . / \omega_{\eta}$ is derived from

$$
\frac{\omega_{. .}}{\omega_{\eta}}=\frac{1}{\varphi_{. .}}\left(\frac{H}{p_{0}}\right) /\left(\frac{H}{p_{0}}\right)^{*},\left(\frac{H}{p_{0}}\right)^{*}=\frac{\hbar}{g \mu_{\mathrm{N}} \eta} .
$$

In this way $\omega_{\mathrm{b}} / \omega_{\eta}$ and $\omega_{\mathrm{T}} / \omega_{\eta}$ are determined and then $\left|\omega_{\eta \mathrm{T}}\right| / \omega_{\eta}=\sqrt{5}\left|\omega_{\mathrm{bt}}\right| / \omega_{\eta}=\sqrt{A_{\eta \mathrm{T}} \omega_{\mathrm{T}} / \omega_{\eta}}$ is obtained.

The signs $s_{\mathrm{t}}$ of $\omega_{\mathrm{bt}}$ (and $\omega_{\eta \mathrm{T}}$ ) and $s_{\mathrm{rt}}$ of $a_{\mathrm{rbt}}$ could be extracted from measurements of flow and heatflow birefringence respectively ${ }^{3,4}$. Experiments on flow birefringence done by Baas 26 showed that $s_{\mathrm{t}}=+1$ for the gases under investigation, i.e. $\mathrm{H}_{2}$, $\mathrm{HD}, \mathrm{CO}, \mathrm{CO}_{2}$ and $\mathrm{N}_{2}$. If both $s_{\mathrm{t}}$ and $s_{\mathrm{rt}}$ are known the quantity $\omega_{\mathrm{br}} / \omega_{\eta}$ is uniquely determined according to Eqs. (2.10), (2.15) by

$$
\begin{aligned}
& \frac{\omega_{\mathrm{br}}}{\omega_{\eta}}=\frac{\omega_{\mathrm{r}}}{\omega_{\eta}} \sqrt{\frac{\omega_{\mathrm{b}}}{\omega_{\mathrm{t}}}}\left(1-\frac{\omega_{\mathrm{tr}}}{\omega_{\mathrm{t}}}\right)^{-1} \\
& {\left[\sqrt{\left.\frac{3}{5} \frac{\lambda_{\mathrm{iso}}}{\lambda_{\text {iso }}^{\mathrm{t}}}\left(1-A_{\mathrm{tr}}\right) a_{\mathrm{rbt}}-\frac{\omega_{\mathrm{bt}}}{\sqrt{\omega_{\mathrm{b}} \omega_{\mathrm{t}}}}\left(1-\frac{\omega_{\mathrm{tr}}}{\omega_{\mathrm{r}}}\right)\right] .}\right.}
\end{aligned}
$$

The sign $s_{\mathrm{r}}$ of $\omega_{\mathrm{br}}$ is equal to that of $a_{\mathrm{rbt}}$ for gases (like $\mathrm{HD}, \mathrm{CH}_{4}, \mathrm{CO}, \mathrm{N}_{2}$ ) where

$$
\sqrt{\frac{3}{5} \frac{\lambda_{\text {iso }}^{\mathrm{t}}\left(1-A_{\mathrm{tr}}\right)}{\lambda_{\text {iso }}}}\left|a_{\mathrm{rbt}}\right|>\frac{\left|\omega_{\mathrm{bt}}\right|}{\sqrt{\omega_{\mathrm{b}} \omega_{\mathrm{t}}}}\left(1-\frac{\omega_{\mathrm{tr}}}{\omega_{\mathrm{r}}}\right)
$$

applies,

$$
s_{\mathrm{r}}=s_{\mathrm{rt}} .
$$

If the signs $s_{\mathrm{t}}$ and $s_{\mathrm{rt}}$ are not known there are two possible values for $\left|\omega_{\mathrm{br}}\right|$ according to the relative sign $s_{\mathrm{t}} s_{\mathrm{rt}}$. The quantity $\omega_{\mathrm{br}}$ can only be calculated if the constants $\omega_{\eta}, \omega_{t}, \omega_{\mathrm{r}}, \omega_{\mathrm{tr}}$ are known.

For a determination of these "classical" relaxation constants the field free transport coefficients $\eta$ and $\lambda$ can be replaced by their isotropic values $\eta_{\text {iso }}, \lambda_{\text {iso }}$. Then $\omega_{\eta}$ is given by $(2.5) \omega_{\eta}=p_{0} / \eta$. Often the cross sections $\sigma .$. ,

$$
\omega . .=n_{0} \sqrt{8 k T_{0} /\left(\pi M_{\mathrm{r}}\right)} \sigma . .,
$$

are used instead of the relaxation constants $\omega_{\text {.. }}$; $M_{\mathrm{r}}=M / 2$ is the reduced mass of a pair of molecules. Here, values for $\omega_{\eta}, \sigma_{\eta}$ and $\omega_{. .} / \omega_{\eta}=\sigma . . / \sigma_{\eta}$ will be given.

If data for the bulk viscosity $\eta_{\mathrm{v}}$ are available the ratio $\omega_{0} / \omega_{\eta}$ is calculated from $(2.3)$, viz. $\omega_{0} / \omega_{\eta}=$ $\frac{2}{3} c_{\mathrm{r}}^{2} \eta / \eta_{\mathrm{V}}$, and via the relations (1.17), (1.20) $\omega_{\mathrm{tr}}$ and $\omega_{\mathrm{t}}$ are then known:

$$
-\frac{\omega_{\mathrm{tr}}}{\omega_{\eta}}=\frac{5}{6} \frac{c_{\mathrm{t}}}{c_{\mathrm{r}}} \frac{\omega_{0}}{\omega_{\eta}}, \frac{\omega_{\mathrm{t}}}{\omega_{\eta}}=\frac{2}{3}+\frac{5}{6} \frac{\omega_{0}}{\omega_{\eta}} .
$$

Now, data on thermal conductivity can be used to find values for $\omega_{\mathrm{r}} / \omega_{\eta}$. From Eqs. (2.8), (2.13) and $\lambda_{\text {iso }} \approx \lambda$ one gets 


$$
\begin{gathered}
\frac{\omega_{\mathrm{r}}}{\omega_{\eta}}=\frac{e^{\prime} \omega_{\mathrm{t}} / \omega_{\eta}+2 e^{\prime}\left(-\omega_{\operatorname{tr}} / \omega_{\eta}\right)+\left(\omega_{\mathrm{tr}} / \omega_{\eta}\right)^{2}}{\omega_{\mathrm{t}} / \omega_{\eta}-e^{\prime}}, \\
e^{\prime} \equiv \frac{5}{2} \frac{k}{M} \lambda .
\end{gathered}
$$

This procedure has recently been used by Moraal and McCourt 27 for a determination of relaxation coefficients for the hydrogen isotopes. In this paper the gases $\mathrm{HD}, \mathrm{CH}_{4}, \mathrm{CO}$ and $\mathrm{N}_{2}$ will be considered at the temperature $T_{0}=293 \mathrm{~K}$. The classical relaxation constants are listed in Table 1 , in Table 2 the constants for the tensor polarization and its flux are given.

\section{Boundary Conditions}

Between the hydrodynamic regime and the Knudsen regime transport phenomena in gases can be described by a set of macroscopic variables for which differential equations and boundary conditions are at hand. Differential equations for the mean velocity $\boldsymbol{v}$, the temperatures $T$ and $T^{\prime}$ and the tensor polarization a have been stated in the first part of this paper, the second part deals with boundary conditions. A general thermodynamic method for the derivation of phenomenological boundary conditions is due to Waldmann ${ }^{9}$. This method shall here be applied to a system consisting of a polyatomic gas and a solid body.

\section{$\S 5$. The entropy flux}

The state of the gas is characterized, as in part I, by the following quantities: density $n$, translational and rotational temperatures $T_{\text {trans }}$ and $T_{\text {rot }}$ (or $T$ and $T^{\prime}$ ), mean velocity $\boldsymbol{v}$, translational and rotational heat fluxes $\boldsymbol{q}_{\text {trans }}$ and $\boldsymbol{q}_{\text {rot }}$ (or $\boldsymbol{q}$ and $\boldsymbol{q}^{\prime}$ ), frictional pressure tensor $\overline{\mathbf{p}}$, tensor polarization a and tensor polarization flux $\underline{\mathbf{b}}$. The solid body shall be at rest, its state shall be described by the temper- ature $T_{\text {solid }}$ and the heat flux $\boldsymbol{q}_{\text {solid }}$. The closed interface $\sigma$ between the gas and the solid is assumed to bear no mass, momentum, energy or entropy per unit area. It shall be permeable to fluxes of these quantities with the exception of mass. Hence the continuity of normal velocity at $\sigma$ is required, i.e. for a resting solid

$$
\boldsymbol{v} \cdot \boldsymbol{n}=0
$$

applies; $\boldsymbol{n}$ denotes the outer unit normal of the gas.

Due to the underlying assumption the total entropy of the system is a sum of the entropies of the gas and the solid without any contribution from the interface:

$$
S=\int_{\text {gas }} \varrho s \mathrm{~d} \tau+\int_{\text {solid }}(\varrho s)_{\text {solid }} \mathrm{d} \tau .
$$

The local entropy per unit mass $s$ obeys the equation

$$
\varrho((\partial s / \partial t)+\boldsymbol{v} \cdot \boldsymbol{\nabla} s)+\boldsymbol{\nabla} \cdot \boldsymbol{s}=\Omega,
$$

where $\boldsymbol{s}$ is the local entropy flux and $\Omega$ is the entropy production per unit volume in the gas. Then the total entropy production of the system

$$
\mathrm{d} S / \mathrm{d} t=\vartheta+\vartheta_{\text {solid }}+\Theta
$$

is a sum of the entropy productions within the gas

within the solid $\int_{\text {gas }} \Omega \mathrm{d} \tau$,

$$
\vartheta_{\text {solid }}=\int_{\text {solid }}\left(\boldsymbol{q} \cdot \boldsymbol{\nabla} T^{-1}\right)_{\text {solid }} \mathrm{d} \tau
$$

and of the entropy production at the interface

$$
\Theta=-\int_{\boldsymbol{\sigma}} \boldsymbol{n} \cdot\left(\boldsymbol{s}-\boldsymbol{s}_{\mathrm{solid}}\right) \mathrm{d} \sigma .
$$

This interfacial entropy production is due to a dis-

\begin{tabular}{|c|c|c|c|c|c|c|c|c|c|c|}
\hline & $\frac{\lambda}{10^{+4} \mathrm{erg}}$ & $\frac{\eta_{10^{-4} \mathrm{~g}}}{\mathrm{~cm} \mathrm{~s}}$ & $\frac{\eta_{\mathrm{v}}}{10^{-4} \mathrm{~g}}$ & $\begin{array}{c}\omega_{\eta} \\
10^{10} \\
\mathrm{~s}\end{array}$ & $\begin{array}{l}\sigma_{\eta} \\
\mathrm{A}^{2}\end{array}$ & $\frac{\omega_{0}}{\omega_{\eta}}$ & $\frac{\omega_{\mathrm{t}}}{\omega_{\eta}}$ & $\frac{-\omega_{\mathrm{tr}}}{\omega_{\eta}}$ & $\frac{\omega_{\mathrm{r}}}{\omega_{\eta}}$ & $A_{\mathrm{tr}}$ \\
\hline $\mathrm{HD}$ & $1.52928 \mathrm{a}$ & $1.053^{28 b}$ & $2.56^{27}$ & 0.96 & 19.0 & 0.04 & 0.70 & 0.06 & 1.58 & 0.003 \\
\hline $\mathrm{CH}_{4}$ & $0.341^{28 c}$ & $1.104^{28 c}$ & $1.44^{29}$ & 0.92 & 41.7 & 0.13 & 0.77 & 0.11 & 1.08 & 0.014 \\
\hline $\mathrm{CO}$ & $0.249^{28 c}$ & $1.732^{28 c}$ & $0.93^{29}$ & 0.59 & 35.1 & 0.20 & 0.83 & 0.25 & 1.96 & 0.038 \\
\hline $\mathrm{N}_{2}$ & $0.256^{28 c}$ & $1.758^{28 c}$ & $1.28^{29}$ & 0.58 & 34.6 & 0.15 & 0.79 & 0.18 & 1.90 & 0.022 \\
\hline
\end{tabular}
continuity of the normal entropy fluxes at $\sigma$.

In the wellknown theory on thermodynamics of irreversible processes the entropy production $\vartheta$ within the gas is used as starting point for the derivation of linear constitutive laws, e.g. for the friction pressure tensor and the heat flux. For

Table 1. Classical relaxation constants calculated from $\lambda, \eta, \eta_{\mathrm{v}}$ at $T_{0}=293 \mathrm{~K}$; the value for $\omega_{\eta}$ refers to $p_{0}=1 \mathrm{~atm}$. 
simple fluids the same procedure has been applied to the interfacial entropy production $\Theta$ by L. Waldmann ${ }^{9}$ in order to find phenomenological boundary conditions. The application of this method requires the knowledge of the entropy fluxes. In the solid one simply has

$$
\boldsymbol{s}_{\text {solid }}=\left(\boldsymbol{q}^{-1}\right)_{\text {solid }},
$$

the entropy flux within the gas is computed by use of the distribution function $f$ for the nonequilibrium state. The ansatz

$$
s=-\frac{k}{M}\left\langle\log \frac{1}{e}\left(\frac{h}{M}\right)^{3} f\right\rangle
$$

for the specific entropy leads to the following expression for the entropy flux

$$
\boldsymbol{s}=-n k\left\langle(\boldsymbol{c}-\boldsymbol{v}) \log \frac{1}{e}\left(\frac{h}{M}\right)^{3} f\right\rangle .
$$

In the same approximation for $f$ which had been used for the derivation of the transport relaxation Eqs. (1.3)-(1.11) the entropy flux is calculated up to terms quadratic in the moments. The result is ${ }^{12}$ :

$$
\begin{aligned}
\boldsymbol{s}= & \boldsymbol{q}_{\text {trans }} T_{\text {trans }}^{-1}+\boldsymbol{q}_{\text {rot }} T_{\text {rot }}^{-1} \\
& -\frac{2}{5}\left(T_{0} p_{0}\right)^{-1} \overline{\mathbf{p}} \cdot \boldsymbol{q}_{\text {trans }}-n_{0} k\left(c_{0} / / \sqrt{3}\right) \underline{\mathbf{b}}: \mathbf{a} \\
\approx & \boldsymbol{q} T^{-1}-T_{0}^{-2} \boldsymbol{q}^{\prime} T^{\prime} \\
& -\frac{2}{5}\left(T_{0} p_{0}\right)^{-1} \overline{\mathbf{p}} \cdot \boldsymbol{q}_{\text {trans }}-n_{0} k\left(c_{0} / / 3\right) \underline{\mathbf{b}}: \mathbf{a} .
\end{aligned}
$$

In a polyatomic gas the translational and rotational degrees of freedom contribute to the entropy flux, viz. $\boldsymbol{q}_{\text {trans }} T_{\text {trans }}^{-1}$ and $\boldsymbol{q}_{\text {rot }} T_{\text {rot }}^{-1}$ respectively. The third term in (5.8), $-\frac{2}{5}\left(T_{0} p_{0}\right)^{-1} \overrightarrow{\mathbf{p}} \cdot \boldsymbol{q}_{\text {trans }}$ is typical for the rarefied gas regime. The factor $\frac{2}{5} p_{0}{ }^{-1}$ also shows up in the Burnett terms occurring in the constitutive laws (2.4), (2.6) for the friction pressure tensor $\left[\sim \frac{2}{5} p_{0}^{-1} \boldsymbol{\nabla}_{\text {trans }}\right]$ and the translational heat flux $\left[\sim \frac{2}{5} p_{0}{ }^{-1} \nabla \cdot \overline{\mathbf{p}}\right]$, respectively. A further contribution to the entropy flux is due to the existence of collisional induced alignments in the gas which are here represented by the tensor polarization and its flux. This term will finally supply us with boundary conditions for the tensor polarization.

\section{$\S 6$. The interfacial entropy production}

With the expressions (5.6) and (5.8) for the entropy fluxes the interfacial entropy production $\Theta$ (5.5) is rewritten as

$$
\begin{aligned}
\Theta & =\int_{\sigma} \mathrm{d} \sigma\left[-\left(\boldsymbol{q} T^{-1}-\boldsymbol{q}_{\mathrm{solid}} T_{\mathrm{solid}}^{-1}\right) \cdot \boldsymbol{n}+T_{0}^{-1} \boldsymbol{q}^{\prime} \cdot \boldsymbol{n} T^{\prime}\right. \\
& \left.+\frac{2}{5}\left(T_{0} p_{0}\right)^{-1} \boldsymbol{n} \cdot \overline{\mathbf{p}} \cdot \boldsymbol{q}_{\mathrm{trans}}+n_{0} k c_{0} / \sqrt{3}(\boldsymbol{n} \cdot \underline{\mathbf{b}}): \mathbf{a}\right] .
\end{aligned}
$$


The integrand in (6.1) shall be transformed into a sum over bilinear products of independent "fluxes" and "forces". Then linear relations between "fluxes" and "forces" are set up, and these are the boundary conditions.

The normal heat fluxes $\boldsymbol{q} \cdot \boldsymbol{n}$ and $\boldsymbol{q}_{\text {solid }} \cdot \boldsymbol{n}$ are not independent of each other, they are interrelated through a condition which derives from the conservation of the total energy of the system ${ }^{9}$ :

$$
E=\int_{\text {gas }} \varrho e_{g} \mathrm{~d} \tau+\int_{\text {solid }} \varrho_{\mathrm{s}} e_{\mathrm{S}} \mathrm{d} \tau
$$

For the local specific energy per unit mass in the gas the following exact equation is obtained from the kinetic equation

$$
\varrho\left(\partial e_{g} / \partial t+\boldsymbol{v} \cdot \boldsymbol{\nabla} e_{g}\right)=-\boldsymbol{\nabla} \cdot(\mathbf{p} \cdot \boldsymbol{v}+\boldsymbol{q})
$$

for the solid we simply have

$$
\varrho_{\mathrm{s}}\left(\partial \varrho_{\mathrm{s}} / \partial t\right)=-\boldsymbol{\nabla} \cdot \boldsymbol{q}_{\mathrm{solid}}
$$

The conservation of total energy

$\mathrm{d} E / \mathrm{d} t=0=\int_{\text {gas }} \varrho\left(\partial e_{g} / \partial t+\boldsymbol{v} \cdot \boldsymbol{\nabla} e_{g}\right) \mathrm{d} \tau+\int_{\text {solid }} \varrho_{\mathrm{S}}\left(\partial e_{\mathrm{S}} / \partial t\right) \mathrm{d} \tau$ then reduces to an integral condition for the energy at the interface, viz.

$$
\int_{\sigma} \mathrm{d} \sigma\left[\left(\boldsymbol{q}-\boldsymbol{q}_{\mathrm{solid}}\right) \cdot \boldsymbol{n}+\boldsymbol{k} \cdot \boldsymbol{v}\right]=0 .
$$

It states that overall the heat produced at $\sigma$ by friction is balanced by a discontinuity of the normal heat fluxes. The local force the gas exerts per unit area of the solid is denoted by $\boldsymbol{k}=\mathbf{p} \cdot \boldsymbol{n}$. Due to (5.1) only the frictional force plays a role in $\boldsymbol{k} \cdot \boldsymbol{v}$ :

$$
\boldsymbol{k}^{\tan } \cdot \boldsymbol{v}^{\tan }=(\overline{\mathbf{p}} \cdot \boldsymbol{n})^{\tan } \cdot \boldsymbol{v}^{\tan }=\boldsymbol{k} \cdot \boldsymbol{v} .
$$

The tangential component of the vector $\boldsymbol{k}$ is given by $\boldsymbol{k}^{\tan }=\boldsymbol{k}-\boldsymbol{n}(\boldsymbol{n} \cdot \boldsymbol{k})$.

By introduction of a "surface heat flux" $\boldsymbol{Q}$ $(\boldsymbol{Q} \cdot \boldsymbol{n}=0)$ the overall condition (6.2) is transformed into a local one ${ }^{9}$ :

$$
\left(\boldsymbol{q}-\boldsymbol{q}_{\mathrm{solid}}\right) \cdot \boldsymbol{n}+\boldsymbol{k}^{\tan } \cdot \boldsymbol{v}^{\tan }=\boldsymbol{\nabla}^{\tan } \cdot \boldsymbol{Q} .
$$

According to $\nabla^{\tan } \cdot \boldsymbol{Q}=\frac{1}{\sqrt{G}} \frac{\partial}{\partial \xi^{i}}\left(\sqrt{G} \boldsymbol{X}^{i} \cdot \boldsymbol{Q}\right)$ and $\mathrm{d} \sigma=\sqrt{G} \mathrm{~d} \xi^{1} \mathrm{~d} \xi^{2}$ Eq. (6.2) really follows from (6.3) by integration over the closed surface $\sigma$. The quantities $\xi^{1}, \xi^{2}$ are parameters which characterize the interface $\sigma, \boldsymbol{X}^{i}(i=1,2)$ are the tangential basis vectors ${ }^{9}$. By general considerations Waldmann showed ${ }^{9}$ that the surface heat flux $\boldsymbol{Q}$ is Galilean invariant.

Now, the first term in (6.1) is rewritten by use of the identity

$$
\boldsymbol{n} \cdot\left(\boldsymbol{q} T^{-1}-\boldsymbol{q}_{\text {solid }} T_{\text {solid }}^{-1}\right)=\frac{1}{2} \boldsymbol{n} \cdot\left(\boldsymbol{q}+\boldsymbol{q}_{\text {solid }}\right)\left(T^{-1}-T_{\text {solid }}^{-1}\right)+\boldsymbol{n} \cdot\left(\boldsymbol{q}-\boldsymbol{q}_{\text {solid }}\right) \frac{1}{2}\left(T^{-1}+T_{\text {solid }}^{-1}\right)
$$

and of (6.3). Up to terms quadratic in deviations from equilibrium we have

$$
\begin{aligned}
-\boldsymbol{n} \cdot\left(\boldsymbol{q} T^{-1}-\boldsymbol{q}_{\text {solid }} T_{\text {solid }}^{-1}\right) & \approx \frac{1}{2} \boldsymbol{n} \cdot\left(\boldsymbol{q}+\boldsymbol{q}_{\mathrm{solid}}\right)\left(T-T_{\text {solid }}\right) T_{0}^{-2} \\
& +T_{0}^{-1} \boldsymbol{k}^{\tan } \cdot \boldsymbol{v}^{\tan }-T_{0}^{-2} \boldsymbol{Q} \cdot \boldsymbol{\nabla}^{\tan } \frac{1}{2}\left(T+T_{\text {solid }}\right)-\boldsymbol{\nabla}^{\tan } \cdot\left[\boldsymbol{Q} \frac{1}{2}\left(T^{-1}+T_{\text {solid }}^{-1}\right)\right],
\end{aligned}
$$

the last term vanishes upon integration over the closed surface $\sigma$. In a similar way the third term in Eq. (6.1) is treated:

$$
\boldsymbol{n} \cdot \overline{\mathbf{p}} \cdot \boldsymbol{q}_{\mathrm{trans}}=\boldsymbol{n} \cdot \overline{\mathbf{p}} \cdot \boldsymbol{n}\left(\boldsymbol{n} \cdot \boldsymbol{q}_{\mathrm{trans}}\right)+(\boldsymbol{n} \cdot \overline{\mathbf{p}})^{\tan } \cdot\left(\boldsymbol{q}_{\operatorname{trans}}\right)^{\tan }=(\boldsymbol{n} \cdot \overline{\mathbf{p}} \cdot \boldsymbol{n})\left(c_{\mathrm{t}} \boldsymbol{n} \cdot \boldsymbol{q}+\boldsymbol{n} \cdot \boldsymbol{q}^{\prime}\right)+\boldsymbol{k}^{\tan } \cdot\left(\boldsymbol{q}_{\mathrm{trans}}\right)^{\tan } \cdot
$$

For $\boldsymbol{n} \cdot \boldsymbol{q}=\frac{1}{2} \boldsymbol{n} \cdot\left(\boldsymbol{q}+\boldsymbol{q}_{\mathrm{solid}}\right)+\frac{1}{2} \boldsymbol{n} \cdot\left(\boldsymbol{q}-\boldsymbol{q}_{\text {solid }}\right)$ again (6.3) is used. If terms which are of third order in the macroscopic variables are neglected the interfacial entropy production (6.1) is given by:

$$
\begin{aligned}
& \Theta=\int_{\sigma} \mathrm{d} \sigma T_{0}{ }^{-1}\left[\frac{1}{2} \boldsymbol{n} \cdot\left(\boldsymbol{q}+\boldsymbol{q}_{\text {solid }}\right)\left(T-T_{\text {solid }}+\frac{2}{5} c_{\mathrm{t}} \frac{T_{0}}{p_{0}} k_{n}{ }^{\prime}\right) T_{0}{ }^{-1}\right. \\
& +\boldsymbol{n} \cdot \boldsymbol{q}^{\prime}\left(T^{\prime}+\frac{2}{5} \frac{T_{0}}{p_{0}} k_{n}{ }^{\prime}\right) T_{0}{ }^{-1}+\boldsymbol{k}^{\tan } \cdot\left(\boldsymbol{v}+\frac{2}{5} p_{0}^{-1} \boldsymbol{q}_{\mathrm{trans}}\right)^{\tan } \\
& \left.-T_{0}{ }^{-1} \boldsymbol{Q} \cdot \nabla^{\tan \frac{1}{2}}\left(T+T_{\text {solid }}+\frac{2}{5} c_{\mathrm{t}} \frac{T_{0}}{p_{0}} k_{n}{ }^{\prime}\right)+\frac{p_{0} c_{0}}{\sqrt{3}}(\boldsymbol{n} \cdot \underline{\mathbf{b}}): \mathbf{a}\right] .
\end{aligned}
$$

The following abbreviations have been used:

$$
\boldsymbol{k}=\mathbf{p} \cdot \boldsymbol{n}, \quad k_{n}{ }^{\prime}=\boldsymbol{n} \cdot \overline{\mathbf{p}} \cdot \boldsymbol{n} .
$$


Finally, the second rank tensors $\mathbf{a}$ and $\boldsymbol{n} \cdot \underline{\mathbf{b}}$ are decomposed into its independent surface components, namely the scalars $a$ and $b$

$$
a=\boldsymbol{n} \cdot \mathbf{a} \cdot \boldsymbol{n}, \quad b=(\boldsymbol{n} \cdot \underline{\mathbf{b}}):(\boldsymbol{n} \boldsymbol{n}),
$$

the tangential vectors $\boldsymbol{a}$ and $\boldsymbol{b}$

$$
\boldsymbol{a}=(\mathbf{a} \cdot \boldsymbol{n})^{\tan }, \quad \boldsymbol{b}=(\boldsymbol{n} \cdot \underline{\mathbf{b}} \cdot \boldsymbol{n})^{\tan ,}
$$

and the symmetric traceless second rank surface tensors $\mathfrak{a}$ and $\mathfrak{B}$ with

$$
\mathfrak{a} \cdot \boldsymbol{n}=\mathbf{0}=\boldsymbol{n} \cdot \mathfrak{a}, \quad \boldsymbol{n} \cdot \mathfrak{B}=\mathbf{0}=\mathfrak{B} \cdot \boldsymbol{n} .
$$

The decomposition

$$
\begin{aligned}
\mathbf{a} & =a \frac{3}{2} \overline{\boldsymbol{n} \boldsymbol{n}}+2 \overrightarrow{\boldsymbol{a} \boldsymbol{n}}+\mathfrak{a}, \\
\boldsymbol{n} \cdot \underline{\mathbf{b}} & =b \frac{3}{2} \overline{\boldsymbol{n} \boldsymbol{n}}+2 \overrightarrow{\boldsymbol{b} \boldsymbol{n}}+\mathfrak{B}
\end{aligned}
$$

may be regarded as definition of $\mathfrak{a}$ and $\mathfrak{B}$ respectively. Due to the relation

$$
(\boldsymbol{n} \cdot \underline{\mathbf{b}}): \mathbf{a}=\frac{3}{2} b a+2 \boldsymbol{b} \cdot \boldsymbol{a}+\boldsymbol{B}: \mathfrak{a}
$$

the interfacial entropy production, cf. Eq. (6.4), splits up into three terms

$$
\Theta=\int_{\sigma} \mathrm{d} \sigma T_{0}^{-1}\left(I_{S}+I_{\mathrm{V}}+I_{\mathrm{T}}\right) .
$$

These terms consist of products of surface scalars

$$
\begin{aligned}
& I_{S}=T_{0}{ }^{-1}\left(T-T_{\text {solid }}+\frac{2}{5} c_{\mathrm{t}} \frac{T_{0}}{p_{0}} k_{n}{ }^{\prime}\right) \frac{1}{2}\left(\boldsymbol{q}+\boldsymbol{q}_{\mathrm{solid}}\right) \cdot \boldsymbol{n} \\
& +T_{0}{ }^{-1}\left(T^{\prime}+\frac{2}{5} \frac{T_{0}}{p_{0}} k_{n}{ }^{\prime}\right) \boldsymbol{q}^{\prime} \cdot \boldsymbol{n}+\frac{p_{0} c_{0}}{\sqrt{3}} \frac{3}{2} a b,(6.12)
\end{aligned}
$$

of tangential vectors

$$
\begin{aligned}
I_{\mathrm{V}}= & \left(\boldsymbol{v}+\frac{2}{5} p_{0}^{-1} \boldsymbol{q}_{\mathrm{trans}}\right)^{\tan } \cdot \boldsymbol{k}^{\tan } \\
& -T_{0}{ }^{-1} \boldsymbol{Q} \cdot \nabla^{\tan \frac{1}{2}}\left(T+T_{\mathrm{solid}}+\frac{2}{5} c_{\mathrm{t}} \frac{T_{0}}{p_{0}} k_{n^{\prime}}\right) \\
& +\frac{p_{0} c_{0}}{V_{3}} 2 \boldsymbol{a} \cdot \boldsymbol{b},
\end{aligned}
$$

and of second rank (symmetric traceless) surface tensors

$$
I_{\mathrm{T}}=\frac{p_{0} c_{0}}{\sqrt{3}} \mathfrak{a}: \mathfrak{B} .
$$

Now, the interfacial entropy production $\Theta$ has been written as a sum over bilinear products of independent "fluxes" and "forces", hence it has the proper form for the derivation of boundary conditions.

\section{§ 7. Boundary Conditions}

If the "fluxes" are written as linear functions of the "forces" the interfacial entropy production becomes a quadratic form of the "forces". Since the second law of thermodynamics requires a positive entropy production, $\Theta \geqq 0$ applies, hence the quadratic form has to be positive semidefinite ${ }^{9}$.

The phenomenological coefficients occurring in the boundary conditions could have tensorial character, the tensors being built up from the unit vectors $\boldsymbol{n}$ and $\boldsymbol{h}$ (direction of the magnetic field) and from tensors pertaining to the curvature of the interface $\sigma$. This possibility shall not be considered here, the coefficients are assumed to be scalar quantities. Then there is no coupling between scalar, vectorial and tensorial "fluxes" at the interface.

In this approximation three scalar boundary conditions for $T, T^{\prime}$ and $a$ are derived from $I_{\mathrm{S}}$, furthermore $I_{\mathrm{V}}$ supplies us with three conditions for the tangential vectors $\boldsymbol{v}^{\tan }, \boldsymbol{Q}$ and $\boldsymbol{a}$, and from $I_{\mathrm{T}}$ a condition for the second rank tensor $\mathfrak{a}$ is obtained.

$$
\begin{aligned}
& T-T_{\mathrm{solid}}+\frac{2}{5} c_{\mathrm{t}} \frac{T_{0}}{p_{0}} k_{n}{ }^{\prime}=L_{11}^{(\mathrm{S})} \frac{1}{2}\left(\boldsymbol{q}+\boldsymbol{q}_{\mathrm{solid}}\right) \cdot \boldsymbol{n}+L_{12}^{(\mathrm{S})} \boldsymbol{q}^{\prime} \cdot \boldsymbol{n}+L_{13}^{(\mathrm{S})} \frac{3}{2} \frac{p_{0} c_{0}}{\sqrt{3}} b \\
& T^{\prime}+\frac{2}{5} \frac{T_{0}}{p_{0}} k_{n}{ }^{\prime}=L_{21}^{(\mathrm{S})} \frac{1}{2}\left(\boldsymbol{q}+\boldsymbol{q}_{\mathrm{solid}}\right) \cdot \boldsymbol{n}+L_{22}^{(\mathrm{S})} \boldsymbol{q}^{\prime} \cdot \boldsymbol{n}+L_{23}^{(\mathrm{S})} \frac{3}{2} \frac{p_{0} c_{0}}{\sqrt{3}} b \\
& T_{0} a=L_{31}^{(\mathrm{S})} \frac{1}{2}\left(\boldsymbol{q}+\boldsymbol{q}_{\mathrm{solid}}\right) \cdot \boldsymbol{n}+L_{32}^{(\mathrm{S})} \boldsymbol{q}^{\prime} \cdot \boldsymbol{n}+L_{33}^{(\mathrm{S})} \frac{3}{2} \frac{p_{0} c_{0}}{V^{3}} b \\
& \left(\boldsymbol{v}+\frac{2}{5} p_{0}^{-1} \boldsymbol{q}_{\mathrm{trans}}\right)^{\tan }=L_{11}^{(\mathrm{V})} \boldsymbol{k}^{\tan }+L_{12}^{(\mathrm{V})} \nabla^{\tan \frac{1}{2}}\left(T+T_{\text {solid }}+\frac{2}{5} c_{\mathrm{t}} \frac{T_{0}}{p_{0}} k_{n}{ }^{\prime}\right)+L_{13}^{(\mathrm{V})} 2 p_{0} \boldsymbol{b} \\
& -T_{0}{ }^{-1} \boldsymbol{Q}=L_{21}^{(\mathrm{V})} \boldsymbol{k}^{\tan }+L_{22}^{(\mathrm{V})} \nabla^{\tan \frac{1}{2}}\left(T+T_{\text {solid }}+{ }_{5}^{2} c_{\mathrm{t}} \frac{T_{0}}{p_{0}} k_{n}{ }^{\prime}\right)+L_{23}^{(\mathrm{V})} 2 p_{0} \boldsymbol{b}
\end{aligned}
$$




$$
\begin{aligned}
\frac{c_{0}}{\sqrt{3}} \boldsymbol{a} & =L_{31}^{(\mathrm{V})} \boldsymbol{k}^{\tan }+L_{32}^{(\mathrm{V})} \nabla^{\tan \frac{1}{2}}\left(T+T_{\text {solid }}+\frac{2}{5} c_{\mathrm{t}} \frac{T_{0}}{p_{0}} k_{n}{ }^{\prime}\right)+L_{33}^{(\mathrm{V})} 2 p_{0} \boldsymbol{b} \\
\mathfrak{a} & =L_{11}^{(\mathrm{T})} \mathfrak{B} .
\end{aligned}
$$

In addition to these linear phenomenological boundary conditions there are two further conditions, namely the continuity of normal velocities (5.1) and the linearized version of Eq. (6.3) which relates the discontinuity of normal heat fluxes to the surface heat flux:

$$
\begin{gathered}
\boldsymbol{v} \cdot \boldsymbol{n}=0, \\
\left(\boldsymbol{q}-\boldsymbol{q}_{\mathrm{solid}}\right) \cdot \boldsymbol{n}=\boldsymbol{\nabla}^{\tan } \cdot \boldsymbol{Q} .
\end{gathered}
$$

The interfacial entropy production is positive if the quantities $I_{\mathrm{S}}, I_{\mathrm{V}}$ and $I_{\mathrm{T}}$ are positive, i.e. the matrices of the phenomenological coefficients have to be positive semidefinite:

$$
L_{i i}^{(n)} \geqq 0, \quad L_{i i}^{(n)} L_{k k}^{(n)}-L_{i k}^{(n)} L_{k i}^{(n)} \geqq 0,
$$

$i, k=1,2,3$ for $n=\mathrm{S}, \mathrm{V}$ and $i, k=1$ for $n=\mathrm{T}$.

Further restrictions on the coefficients are due to Onsager relations 9 . Since all scalar "forces" have the same time reversal behaviour,

$$
L_{k i}^{(\mathrm{S})}=L_{i k}^{(\mathrm{S})}, \quad i, k=1,2,3
$$

applies. The vectorial "forces" show different time reversal behaviour, hence we have

$L_{21}^{(\mathrm{V})}=L_{12}^{(\mathrm{V})}, L_{31}^{(\mathrm{V})}=-L_{13}^{(\mathrm{V})}, L_{32}^{(\mathrm{V})}=-L_{23}^{(\mathrm{V})}$.

A combination of Eqs. (7.11), (7.12) with Eq. (7.10) results in the inequalities

$$
L_{i i}^{(n)} L_{k k}^{(n)}-\left(L_{i k}^{(n)}\right)^{2} \geqq 0,
$$

$i, k=1,2,3$ for $n=\mathrm{S}$ and $i, k=1,2$ for $n=\mathrm{V}$.

They lead to certain restrictions: If, for example, in (7.4) a thermal slip according to

$$
L_{12}^{(\mathrm{V})} \nabla^{\tan \frac{1}{2}}\left(T+T_{\text {solid }}+\frac{2}{5} c_{\mathrm{t}} \frac{T_{0}}{p_{0}} k_{n}{ }^{\prime}\right)
$$

shall be taken into account, i.e. if $L_{12}^{(\mathrm{V})} \neq 0$ is assumed, then the symmetry (7.12) gives $L_{21}^{(\mathrm{V})}=L_{12}^{(\mathrm{V})} \neq 0$, and according to $(7.10),(7.13) L_{11}^{(\mathrm{V})}>0, L_{22}^{(\mathrm{V})}>0$ apply. Consequently, mechanical slip $\left[\sim L_{11}^{(\vec{V})} \boldsymbol{k}^{\tan }\right]$ has to be considered too and the surface heat flux $\boldsymbol{Q}$ cannot be neglected at all. This aspect is of importance for the thermal force problem ${ }^{10}$.

Condition (7.4) shows the different contributions to the tangential slip velocity at the wall, existing in a rarefied polyatomic gas. Two of them also exist in a monatomic gas, namely mechanical slip $\left[\sim L_{11}^{(\mathrm{V})} \boldsymbol{k}^{\mathrm{tan}}\right]$ and thermal slip. Thermal slip consists of two terms,

$$
-\frac{2}{5} p_{0}^{-1} \boldsymbol{q}_{\mathrm{trans}}^{\tan }
$$

and $\quad L_{12}^{(\mathrm{V})} \nabla^{\tan } \frac{1}{2}\left(T+T_{\text {solid }}+\frac{2}{5} c_{\mathrm{t}} \frac{T_{0}}{p_{0}} k_{n}{ }^{\prime}\right)$.

They add up if there is a tangential gradient of temperature near the wall, e.g. in the thermal force problem ${ }^{10}$. Only the first term, $-\frac{2}{5} p_{0}^{-1} \boldsymbol{q}_{\mathrm{trans}}^{\mathrm{tan}}$, is present in the thermomagnetic pressure difference set up 12,13 , since then the temperature gradient is perpendicular to the wall and, due to a magnetic field, the heat flux $\boldsymbol{q}_{\text {trans }}$ has a component parallel to the wall. Notice, that in this case the factor $\frac{2}{5}$ occurs instead of Maxwell's thermal slip factor $\frac{1}{5}$. This is also of importance for the calculation of the contribution of thermal slip to thermomagnetic

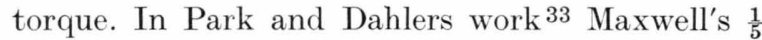
was used.

In a polyatomic gas there is also a polarization induced slip $\sim L_{13}^{(\mathrm{V})} 2 p_{0} \boldsymbol{b}$. The existence of such a "thermomagnetic slip" has been postulated by Waldmann 15 in order to explain slip contributions to thermomagnetic torque. It is also important for a calculation of Knudsen corrections for the Senftleben-Beenakker effect of viscosity 12, 13 and for the theory of thermomagnetic pressure difference ${ }^{12-15}$.

But of equal importance in this connection are the conditions (7.3), (7.6) and (7.7) which determine the value of the tensor polarization at the wall. They summarize mechanisms for the production and destruction of tensor polarization by collisions of the gas molecules with the wall. First let us look at the relations between the surface components of a and $\boldsymbol{n} \cdot \mathbf{b}$ in Eqs. (7.3), (7.6), (7.7). A Kagan polarization $\mathbf{b}$ is set up in a heat conducting gas by collisions of the molecules with each other [see the term $\boldsymbol{a}^{\mathrm{rt}}$ in (2.16)], then by gas-wall collisions it can be transferred into a tensor polarization. On the other hand a tensor polarization is present in a streaming gas [see (3.1)], it can be partially destroyed by gas-wall interaction.

Thus the coefficients $L_{33}^{(\mathrm{S})}, L_{33}^{(\mathrm{V})}, L_{11}^{(\mathrm{T})}$ characterize the transformation of Kagan polarization into ten- 
sor polarization as well as the accomodation of tensor polarization. The remaining terms in (7.3), (7.6) describe direct mechanisms for the production of tensor polarization at the wall by heat fluxes and frictional forces of the gas.

Due to the antisymmetry $L_{31}^{(\mathrm{V})}=-L_{13}^{(\mathrm{V})}$ there is a relation between thermomagnetic slip $\sim L_{13}^{(\mathrm{V})} 2 p_{0} \boldsymbol{b}$ and the production of tensor polarization $\sim L_{31}^{(\mathrm{V})} \boldsymbol{k}^{\tan }$.

A similar relation exists between $(7.6)$ and the ansatz (7.5) for the surface heat flux $\boldsymbol{Q}, L_{32}^{(\mathrm{V})}=-L_{23}^{(\mathrm{V})}$. Here, the term $-T_{0} L_{23}^{(\mathrm{V})} 2 p_{0} \boldsymbol{b}$ is responsible for the dependence of $\boldsymbol{Q}$ on the magnetic field. Insertion of the ansatz (7.5) into (7.9) yields an explicit condition for the discontinuity of the normal heat fluxes at the interface.

The discontinuity of temperature $T$ at $\sigma$ is given by Equation (7.1). The temperature jump is due to normal heat fluxes, to a normal frictional force $\left(k_{n}{ }^{\prime}\right)$ and to normal components of the tensor polarization flux. Quite similar terms occur in Eq. (7.2) which determines the value of the difference temperature $T$ " at the wall. In the "isotropic approximation" where the alignments $\mathbf{a}$ and $\mathbf{b}$ are neglected the differential equation (3.12) states that $T^{\prime}$ decays from its value at the interface over a mean free path $L_{\mathrm{T}}{ }^{\prime}$ to $T^{\prime \prime}=0$ within the gas.

An inspection of the boundary conditions shows that the translational temperature always enters via the combination $T_{\text {trans }}+\frac{2}{5} T_{0} p_{0}{ }^{-1} k_{n}{ }^{\prime}$, and that the tangential velocity always occurs in connection with the translational heat flux as

$$
\left(\boldsymbol{v}+\frac{2}{5} p_{0}^{-1} \boldsymbol{q}_{\text {trans }}\right)^{\tan } .
$$

This is due to the term $-\frac{2}{5}\left(T_{0} p_{0}\right)^{-1} \hat{\mathbf{p}} \cdot \boldsymbol{q}_{\text {trans }}$ in the entropy flux (5.8). It should be noticed that the same combination of $\boldsymbol{v}$ and $\boldsymbol{q}_{\text {trans }}$ shows up in the ansatz (2.4) for the friction pressure tensor $\overrightarrow{\mathbf{p}}$. Furthermore, $\boldsymbol{\nabla} T_{\text {trans }}$ and $\frac{2}{5} T_{0} p_{0}{ }^{-1} \boldsymbol{\nabla} \cdot \overline{\mathbf{p}}$ occur together in the constitutive laws $(2.6),(2.7)$ for the translational and rotational heat fluxes. This fact shows the intimate connection between constitutive laws and boundary conditions derived by Waldmann's thermodynamic method ${ }^{9}$.

\section{$\S 8$ A simplified set of boundary conditions}

In this paragraph the general boundary conditions (7.1) - (7.7) will be simplified in two respects. First, the three Eqs. (7.3), (7.6), (7.7) are replaced by one condition for the tensor polarization, then the difference temperature $T^{\prime}$ is neglected throughout.
The value of the tensor polarization at the wall is calculated from Eq. (6.9) $a=a \frac{3}{2} \overrightarrow{\boldsymbol{n n}}+2 \overrightarrow{\boldsymbol{a n}}+\mathfrak{a}$ and from Eqs. (7.3), (7.6), (7.7) which are boundary conditions for its surface components $a, \boldsymbol{a}$ and $\mathfrak{a}$. In contradistinction, the solution of the differential Eqs. (3.2) yields the five components $\mathbf{a}^{(m)} \equiv \mathscr{P}(m)$ : a of the tensor polarization. Since, in general, the relation between these two different decompositions of a will be rather complicated five coupled boundary conditions for the $m$-components $\mathbf{a}^{(m)}$ will be obtained. They can be decoupled by the assumption that the three phenomenological coefficients $L_{33}^{(\mathrm{S})}$, $L_{33}^{(\mathrm{V})}$ and $L_{11}^{(\mathrm{T})}$ can be expressed by one (dimensionless) parameter $C_{\mathrm{a}}$ :

$$
\begin{array}{llll}
3 & p_{0} & c_{0} \\
2 & T_{0} & / 3
\end{array} L_{33}^{(\mathrm{S})}=\begin{gathered}
2 / 3 p_{0} \\
c_{0}
\end{gathered} L_{33}^{(\mathrm{V})}=L_{11}^{(\mathrm{T})} \equiv C_{\mathrm{a}} .
$$

According to Halbritter ${ }^{34}$ the difference between the three coefficients

$$
\frac{3}{2} \frac{p_{0}}{T_{0}} \frac{c_{0}}{\sqrt{3}} L_{33}^{(\mathrm{S})}, \quad{ }_{c_{0}}^{2 / 3} p_{0} L_{33}^{(\mathrm{V})} \text { and } L_{11}^{(\mathrm{T})}
$$

is determined by the angular dependent part of the gas-surface interaction potential. Thus it will be small compared to $L_{11}^{(\mathrm{T})}$. By the use of (8.1) Eqs. (7.3), (7.6), (7.7) can be replaced by one boundary condition for a :

$$
\mathbf{a}=\mathfrak{q} \frac{3}{2} \overline{\boldsymbol{n} \boldsymbol{n}}+2 \overline{\boldsymbol{K} \boldsymbol{n}}+C_{\mathrm{a}} \boldsymbol{n} \cdot \underline{\mathbf{b}},
$$

with $q$ and $\boldsymbol{K}$ given by

$$
\begin{aligned}
\mathfrak{q}= & T_{0}{ }^{-1} L_{31}^{(\mathrm{S})} \frac{1}{2}\left(\boldsymbol{q}+\boldsymbol{q}_{\mathrm{solid}}\right) \cdot \boldsymbol{n}+T_{0}{ }^{-1} L_{32}^{(\mathrm{S})} \boldsymbol{q}^{\prime} \cdot \boldsymbol{n}, \\
\boldsymbol{K} & =\frac{\sqrt{3}}{c_{0}} L_{31}^{(\mathrm{V})} \boldsymbol{k}^{\tan } \\
& +\frac{\sqrt{3}}{c_{0}} L_{32}^{(\mathrm{V})} \nabla^{\tan \frac{1}{2}}\left(T+T_{\text {solid }}+\frac{2}{5} c_{\mathrm{t}} \frac{T_{0}}{p_{0}} k_{n}{ }^{\prime}\right) .
\end{aligned}
$$

If the projection tensors $\mathscr{P}(m)$ are applied to $(8.2)$ five decoupled equations for the $m$-components $\mathbf{a}^{(m)}$ of the tensor polarization are obtained since $\mathscr{P}(m):(\boldsymbol{n} \cdot \mathbf{b})$ only contains $\boldsymbol{n} \cdot \boldsymbol{\nabla} \mathbf{a}^{(m)}$ [see (2.16)].

For many prob'ems the difference temperature $T^{\prime}$ is not of crucial importance and may be neglected. Insofar as the theory of thermomagnetic pressure difference is concerned ${ }^{12-15}$ this is achieved by the assumption

$$
L_{21}^{(\mathrm{S})}=L_{22}^{(\mathrm{S})}=L_{23}^{(\mathrm{S})}=0 .
$$

Very often there are no tangential gradients along the interface so that terms like

$$
\nabla^{\tan \frac{1}{2}}\left(T+T_{\text {solid }}+\frac{2}{5} c_{\mathrm{t}}\left(T_{0} / p_{0}\right) k_{n}{ }^{\prime}\right)
$$


vanish, and the surface heat flux is constant, i.e. $\boldsymbol{\nabla}^{\tan } \cdot \boldsymbol{Q}=0$. Then, according to $(7.9)$ the normal heat fluxes are continuous, $\boldsymbol{q}_{\text {solid }} \cdot \boldsymbol{n}=\boldsymbol{q} \cdot \boldsymbol{n}$. This is inserted into Eqs. (7.1) and (7.3).
If all these approximations are used the boundary conditions $(7.1),(7.4),(8.2)$ and $(7.8)$ reduce to the following equations:

$$
\begin{aligned}
& T-T_{\text {solid }}+\frac{2}{5} c_{\mathrm{t}} \frac{T_{0}}{p_{0}} k_{n}{ }^{\prime}=C_{\mathrm{t}} \frac{l}{\lambda_{\text {iso }}} \boldsymbol{q} \cdot \boldsymbol{n} \\
& +T_{0} \zeta C_{\mathrm{ta}}(\boldsymbol{n} \cdot \mathbf{b}): \boldsymbol{n} \boldsymbol{n}, \\
& \left(\boldsymbol{v}+\frac{2}{5} p_{0}^{-1} \boldsymbol{q}_{\text {trans }}\right)^{\tan }= \\
& C_{\mathrm{m}} \frac{l}{\eta_{\text {iso }}} \boldsymbol{k}^{\tan }+{ }_{\sqrt{3}}^{c_{0}} C_{\mathrm{ma}}(\boldsymbol{n} \cdot \mathbf{b} \cdot \boldsymbol{n})^{\tan }, \\
& \mathbf{a}=C_{\text {at }} \frac{l}{T_{0} \lambda_{\text {iso }}} \boldsymbol{q} \cdot \boldsymbol{n} \frac{3}{2} \overline{\boldsymbol{n} \boldsymbol{n}}+C_{\mathrm{am}} p_{0}^{-1} \overline{\boldsymbol{k}^{\tan \boldsymbol{n}}}+C_{\mathrm{a}} \boldsymbol{n} \cdot \underline{\mathbf{b}}, \\
& \boldsymbol{v} \cdot \boldsymbol{n}=0 .
\end{aligned}
$$

For convenience, dimensionless parameters $C$.. have been introduced:

$$
L_{11}^{(\mathrm{S})}=\frac{l}{\lambda_{\text {iso }}} C_{\mathrm{t}}, \quad L_{13}^{(\mathrm{S})}=\frac{l}{\lambda_{\mathrm{iso}}} C_{\mathrm{ta}}, \quad L_{11}^{(\mathrm{V})}=\frac{l}{\eta_{\text {iso }}} C_{\mathrm{m}}, \quad L_{13}^{(\mathrm{V})}=\frac{c_{0}}{2 \sqrt{3} p_{0}} C_{\mathrm{ma}}
$$

with $\quad C_{\mathrm{at}}=C_{\mathrm{ta}}, \quad C_{\mathrm{am}}=-C_{\mathrm{ma}}$;

$l=\frac{2}{5} \sqrt{2}\left(n_{0} \sigma_{\eta}\right)^{-1}$ is the viscosity mean free path. The inequalities (7.10), (7.13) read now

$$
C_{\mathrm{t}}, C_{\mathrm{m}}, C_{\mathrm{a}} \geqq 0, \quad C_{\mathrm{t}} C_{\mathrm{a}}-\zeta C_{\mathrm{at}}^{2} \geqq 0,
$$

with $\zeta=\frac{24}{25} \sqrt{\frac{2}{\pi}} e^{\prime}=0.766 e^{\prime}$

and $e^{\prime}=\frac{5}{2} \frac{k}{M} \frac{\eta}{\lambda}, \quad$ see $(4.15)$.

The parameters $C_{\mathrm{m}}$ and $C_{\mathrm{t}}$ are the wellknown mechanical slip coefficient and the temperature jump coefficient, respectively. They are related to the accommodation coefficients for tangential momentum $\left(\alpha_{\mathrm{m}}\right)$ and energy $\left(\alpha_{\mathrm{e}}\right)$ by 35

$$
\begin{aligned}
C_{\mathrm{m}} & =\frac{2-\alpha_{\mathrm{m}}}{\alpha_{\mathrm{m}}} \frac{5 \pi}{16}, \\
C_{\mathrm{t}} & =\frac{2-\alpha_{\mathrm{e}}}{\alpha_{\mathrm{e}}} \frac{25 \pi}{16} \frac{1}{e^{\prime}\left(1+3 / c_{\mathrm{t}}\right)} .
\end{aligned}
$$

Both, $C_{\mathrm{m}}$ and $C_{\mathrm{t}}$ are of the order of 1 .

The accommodation of tensor polarization is characterized by the coefficient $C_{\mathrm{a}}$, hence its order of magnitude is expected to be 1 . This is confirmed by Halbritter's ${ }^{7}$ calculations and by the values for $C_{\mathrm{a}}$ extracted ${ }^{12,13}$ from the Knudsen corrections for the Senftleben-Beenakker effect of viscosity. The nondiagnonal coefficients $C_{\text {at }}$ and $C_{\text {am }}$ should be much smaller than 1 , say of the order of $a_{\text {rbt }}$ or $\omega_{\eta \mathrm{T}} / \omega_{\eta}$, since they describe the production of tensor polarization at the wall by normal heat fluxes and tangential forces, respectively. By comparison of theory and experiment for the Knudsen corrections and for thermomagnetic pressure difference very small values for $C_{\text {at }}$ and $C_{\text {am }}$ of the order of $10^{-2}$ to $10^{-3}$ are obtained 12,13 . The parameter $C_{\mathrm{ma}}$ (= $-C_{\text {am }}$ ) characterizes thermomagnetic slip. It has been related to the angular dependent part of the gas-wall interaction by Halbritter ${ }^{7}$. For his version of thermomagnetic slip

$$
v_{\mu}^{\tan }=c_{0} C_{\mathrm{S}}\left(b_{\mu, v \lambda} n_{\nu} n_{\lambda}\right)^{\tan }
$$

he calculated a value of $C_{\mathrm{s}}=-0.78 \cdot 10^{-2}$ for HD.

Notice, that here $v_{\mu}^{\tan }=\frac{c_{0}}{V_{3}} C_{\mathrm{ma}}\left(n_{\lambda} b_{\lambda, v \mu} n_{v}\right)^{\tan }$ has been obtained for thermomagnetic slip.

In summary, a set of constitutive laws and differential equations for the macroscopic variables characterizing the state of a polyatomic gas has been derived from the linearized Waldmann-Snider equation. Boundary conditions for some moments of the distribution function have been obtained according to Waldmann's method from the interfacial entropy production. Now, a lot of transpot phenomena occurring in a rarefied polyatomic gas (in the presence of a magnetic field) can be treated. Interesting problems are the Knudsen corrections for the Senftleben-Beenakker effect of viscosity, for flow 
and heat-flow birefringence and a theory of thermomagnetic pressure difference. Details will be presented in subsequent papers.

1 L. Waldmann, Z. Naturforsch. 12a, 660 [1957]; 13 a, 609 [1958]; in Handbuch der Physik (ed. S. Flügge) Vol. 12, Springer, Berlin 1958. - R. F. Snider, J. Chem. Phys. 32, 1051 [1960].

2 For a review see: J. J. M. Beenakker and F. R. McCourt, Ann. Rev. Phys. Chem. 21, 47 [1970].

3 S. Hess, Springer Tracts in Mod. Phys. 54, 136 [1970].

4 S. Hess, in Proc. 7th Int. Symp. Rarefied Gas Dynamics, Pisa 1970, ed. D. Dini, Academic Press, New York (in press); Z. Naturforsch. 28 a, 861 [1973].

5 S. Hess, Z. Naturforsch. 24a, 1675, 1852 [1969]; 25a, $350[1970]$.

6 See for example C. Cercignani, "Mathematical Methods in Kinetic Theory", Plenum Press, New York 1969.

7 J. Halbritter, Thesis "Ableitung einer Randbedingung für die Verteilungsfunktion eines Gases aus linearen rotierenden Molekülen", Erlangen 1972.

8 V. D. Borman, L. A. Maksimov, B. I. Nikolayev, and V. I. Troyan, Zh. Eksp. Teor. Fiz. 64, 526 [1973].

9 L. Waldmann, Z. Naturforsch. 22 a, 1269 [1967].

$10 \mathrm{H}$. Vestner, M. Kübel, and L. Waldmann, in Proc. 7th Int. Symp. Rarefied Gas Dynamics, Pisa 1970, ed. D. Dini, Academic Press, New York (in press).

11 L. Waldmann and R. Rübsamen, Z. Naturforsch. 27 a, 1025 [1972].

12 H. Vestner, Thesis "Transporterscheinungen und Randbedingungen in verdünnten mehratomigen Gasen im Magnetfeld", Erlangen 1973.

13 H. Vestner, Z. Naturforsch. 28 a, 869 [1973].

14 H. Vestner, in Proc. 8th Int. Symp. Rarefied Gas Dynamics, Stanford 1972, ed. K. Karamcheti, Academic Press, New York (in press).

15 L. Waldmann, Z. Naturforsch. 22 a, 1678 [1967]; "Invited Paper" at A. P. S. Meeting, Dallas, March 1970.

16 H. Hulsman, F. G. van Kuik, H. F. P. Knaap, and J. J. M. Beenakker, Physica 57, 522 [1972].

17 G. G. Scott, H. W. Sturner, and R. M. Williamson, Phys. Rev. 158, 117 [1967]; Phys. Letters 25 A, 573 [1967].

18 A. C. Levi and J. J. M. Beenakker, Phys. Letters $25 \mathbf{~ A}$, 350 [1967]. - A. C. Levi, F. R. MeCourt, and J. J. M. Beenakker, Physica 42, 363 [1969].

19 S. Hess and L. Waldmann, Z. Naturforsch. 21 a, 1529 [1966]; 23a, 1893 [1968]. - H. H. Raum and W. E. Köhler, Z. Naturforsch. 25 a, 1178 [1970].

\section{Acknowledgements}

I am indebted very much to Prof. Dr. L. Waldmann for stimulating my interest in these problems and for helpful comments. Thanks are due to my friends and colleagues Univ.-Doz. Dr. S. Hess, Dr. W. E. Köhler and Dr. J. Halbritter for permanent discussions.

20 A. C. Levi, F. R. McCourt, and J. Hajdu, Physica 42, 347 [1969].

21 W. E. Köhler, Thesis "Transporterscheinungen in Gasen aus linearen Molekülen im äußeren Magnetfeld und nichtsphärische intermolekulare Wechselwirkung", Erlangen 1971.

22 E. A. Mason and L. Monchick, J. Chem. Phys. 36, 1622 [1962].

23 S. Hess and L. Waldmann, Z. Naturforsch. 26 a, 1057 [1971].

24 C. S. Wang Chang, G. E. Uhlenbeck, and J. de Boer, in "Studies in Statistical Mechanies", Vol. II, North-Holland, Amsterdam 1964.

25 W. E. Köhler and H. H. Raum, Z. Naturforsch. 27 a, 1383 [1972].

26 F. Baas, Phys. Letters 36 A, 107 [1971]; Physica, to be published.

27 H. Moraal and F. R. McCourt, Physica 60, 73 [1972].

28 a) H. Cauwenberg and W. van Dael, Physica 54, 347 [1971]. b) A. Rietveld and A. van Itterbeek, Physica 23, 838 [1957]. c) Data Book, edited by Thermophysical Properties Research Center, Lafayette, Indiana 1966 , Vol. 2.

29 G. J. Prangsma, Thesis "Relaxation Phenomena in Simple Gases", Leiden 1971. - G. J. Prangsma, A. H. Alberga, and J. J. M. Beenakker, Physica 64, 278 [1973].

30 a) W. E. Quinn, J. M. Baker, J. T. La Tourrette, and N. F. Ramsey, Phys. Rev. 112, 1929 [1958]. b) C. H. Anderson and N. F. Ramsey, Phys. Rev. 149, 14 [1966]. c) I. Ozier, P. N. Yi, A. Khosla, and N. F. Ramsey, J. Chem. Phys. 46, 1530 [1967]. d) S. I. Chan, M. R. Baker, and N. F. Ramsey, Phys. Rev. 136 A, 1224 [1964].

31 J. Korving, Physica 50, 27 [1970]. - H. Hulsman, E. J van Waasdijk, A. L. J. Burgmans, H. F. P. Knaap, and J. J. M. Beenakker, Physica 50, 53 [1970]. - H. Hulsman, F. G. van Kuik, K. W. Walstra, H. F. P. Knaap, and J. J. M. Beenakker, Physica 57, 501 [1972].

32 L. J. F. Hermans, J. M. Koks, A. F. Hengeveld, and H. F. P. Knaap, Physica 50, 410 [1970].

33 W. H. Park and J. S. Dahler, J. Chem. Phys. 56, 1995 [1972].

34 J. Halbritter, private communication.

35 E. H. Kennard, Kinetic Theory of Gases, McGraw-Hill, New York 1938. 